\title{
Source Depth Estimation Using a Horizontal Array by Matched-Mode Processing in the Frequency-Wavenumber Domain
}

\author{
Barbara Nicolas, Jérôme I. Mars, and Jean-Louis Lacoume \\ Laboratoire des Images et des Signaux, 961 Rue de la Houille Blanche, 38402 Saint Martin d'Hères Cedex, BP 46, France
}

Received 20 June 2005; Revised 18 October 2005; Accepted 31 October 2005

Recommended for Publication by Joe C. Chen

In shallow water environments, matched-field processing (MFP) and matched-mode processing (MMP) are proven techniques for doing source localization. In these environments, the acoustic field propagates at long range as depth-dependent modes. Given a knowledge of the modes, it is possible to estimate source depth. In MMP, the pressure field is typically sampled over depth with a vertical line array (VLA) in order to extract the mode amplitudes. In this paper, we focus on horizontal line arrays (HLA) as they are generally more practical for at sea applications. Considering an impulsive low-frequency source $(1-100 \mathrm{~Hz})$ in a shallow water environment (100-400 m), we propose an efficient method to estimate source depth by modal decomposition of the pressure field recorded on an HLA of sensors. Mode amplitudes are estimated using the frequency-wavenumber transform, which is the 2D Fourier transform of a time-distance section. We first study the robustness of the presented method against noise and against environmental mismatches on simulated data. Then, the method is applied both to at sea and laboratory data. We also show that the source depth estimation is drastically improved by incorporating the sign of the mode amplitudes.

Copyright () 2006 Hindawi Publishing Corporation. All rights reserved.

\section{INTRODUCTION}

Passive source localization in shallow water environments has been studied for many decades in underwater acoustics as many sources of interest are present in the ocean: marine mammals, fish, and submarines. These sources emit acoustic waves at different frequencies and localization methods must be adapted to these frequencies. In this paper, we focus on ultra low-frequency waves $(1-100 \mathrm{~Hz})$ which correspond to frequencies emitted by marine mammals or submarines and we estimate source depth.

Beamforming techniques are not suitable for localization in shallow water environments because they do not consider multipath arrivals and complexity of ocean acoustic channels. Matched-field processing (MFP) and matched-mode processing (MMP) constitute, then, alternatives to localize underwater sources.

First proposed by Bucker [1], matched-field processing has been studied extensively in the literature [2-5]. MFP can be seen as a generalized beamforming method which incorporates spatial complexity of acoustic fields in an ocean waveguide. For each source location, the acoustic field received on an array of sensors is simulated. This field is then compared to the pressure field recorded on a real array, using an objective function, which is often defined as the correlation function between real and simulated pressure fields (Bartlett correlator). An overview of these methods is given in [6]. The main drawback of MFP methods is their sensitivity to environmental mismatch due to the use of the global acoustic field.

Matched-mode processing [7-9] is less sensitive to environmental mismatches. This approach uses the property of modal propagation in shallow water waveguides (which has also been extensively used in geoacoustic inversion $[10,11]$ ) and estimates source depth using mode amplitudes (also called mode excitation factors) extracted from real data. Contrary to MFP, matched-mode processing only extracts information about the source location in the pressure field (whereas MFP uses the entire pressure field), which reduces its sensitivity to environmental mismatches. MMP is typically applied to narrow band signals using a vertical line array (VLA) of sensors to extract mode amplitudes.

As information extraction using a horizontal line array (HLA) is generally more adapted to practical applications, we propose a matched-mode method to estimate source depth using an HLA of sensors placed on the sea bottom. 


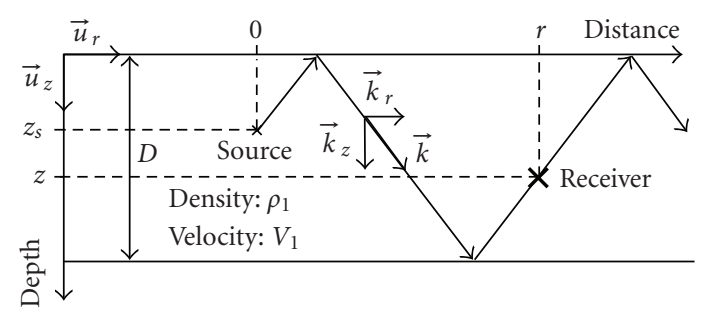

Figure 1: Perfect waveguide.

VLA matched-mode methods cannot be used in this case (as recorded data do not sample the pressure field in depth), so we develop a new method to extract mode amplitudes. Lots of work have been done in geoacoustic inversion to extract mode amplitudes using wavenumbers [12, 13]. In this paper, we develop a simple method to extract mode amplitude. These amplitudes are extracted by modal filtering in the frequency-wavenumber plane $(f-k$ plane $)$ where modes are separated.

After a brief presentation of guided propagation in a shallow water environment and classical matched-mode processing, we develop a matched-mode method of source depth estimation based on the frequency-wavenumber transform. A study of robustness against noise and against environmental mismatch is made. Finally, we validate the proposed method on two real data sets: the first one was recorded in the North Sea where the source depth was roughly known. The second data set was recorded during ultrasonic experiments performed at the Marine Physical Laboratory (SCRIPPS-San Diego) in a perfectly known environment, which allows us to study the error on source depth estimation. We also study the influence of the sign of modal excitation factors and show that its knowledge improves source depth estimation. A future work would consist in estimating this sign on a sea data.

\section{MODES IN AN OCEANIC WAVEGUIDE AND MATCHED-MODE PROCESSING}

\subsection{Normal modes in an oceanic waveguide}

In shallow water environments and for low-frequency waves, the acoustic field can be modeled using normal mode theory. For the sake of simplicity, let us consider a perfect waveguide (Figure 1) made of a homogeneous layer of fluid between perfectly reflecting boundaries at depth 0 (surface) and $D$ (sea bottom). The water layer is characterized by a velocity $V_{1}$ and a density $\rho_{1}$. The study is presented for an omnidirectional harmonic point source, with a frequency $f$ located at depth $z_{s}$ and at range 0 , but results are similar for a broadband source.

Acoustic pressure $P(r, z, t)$ received at $M(r, z)$ can be expressed by $P(r, z, t)=p(r, z) \exp (2 i \pi f t)$, where $p(r, z)$ satisfies the Helmholtz equation [14] and is, at long range, a sum of modes:

$$
p(r, z)=A \sum_{m=1}^{+\infty} \psi_{m}\left(z_{s}\right) \psi_{m}(z) \frac{\exp \left(-2 i \pi k_{r m} r\right)}{\sqrt{k_{r m} r}}
$$

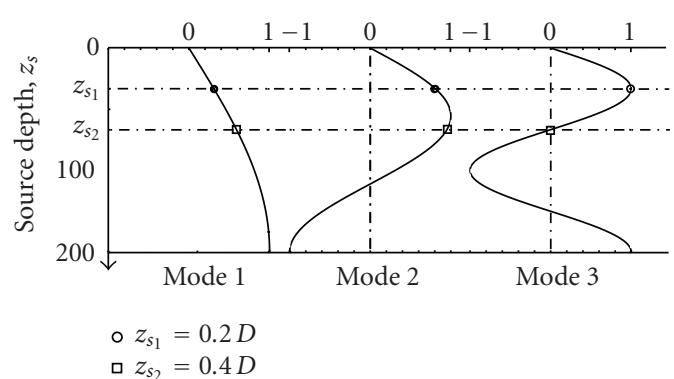

(a)

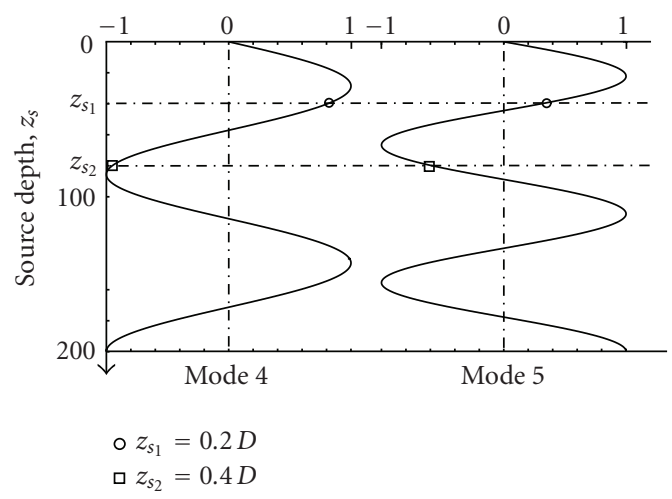

(b)

FIgURE 2: Mode amplitudes (for modes 1 to 5) in a perfect waveguide and examples for two different source depths: $z_{s_{1}}=0.2 D$ and $z_{s_{2}}=0.4 D$.

with $A$ a constant. By homogeneity with the temporal frequency $f, k$ is defined as a spatial frequency $k=f / V_{1}$ and is the inverse of the wavelength (for the sake of simplicity, $k_{r m}$, the horizontal spatial frequency will be called the wavenumber in the following even if there is a factor $2 \pi$ between wavenumber and spatial frequency). The wavenumber spectrum of the modes is discrete and each mode is associated with a unique wavenumber. The mode amplitude $\psi_{m}$, also called mode excitation factor, is a function of the source depth $z_{s}$ :

$$
\psi_{m}\left(z_{s}\right)=\sqrt{\frac{2}{D}} \sin \left(2 \pi k_{z m} z_{s}\right)
$$

with $k_{z m}=(2 m-1) / 4 D$. Figure 2 represents these mode amplitudes, normalized between -1 and 1 , as a function of the source depth for a perfect waveguide $(D=200 \mathrm{~m})$. Two examples at different source depths: $z_{s_{1}}=0.2 D$ (circles) and $z_{s_{2}}=0.4 D$ (squares) are presented.

This short study of propagation in shallow water waveguides shows that mode amplitude is a function of the source depth $z_{s}$. Matched-mode processing (MMP) use this property to localize underwater sources by mode amplitudes extraction. 


\subsection{Classical matched-mode processing (MMP)}

Matched-mode processing (MMP) methods are widely used to localize underwater sources in shallow water environments. These methods estimate source depth [7], distance source receiver [16], or these two parameters jointly $[8,9$, 17].

To estimate the source depth, matched-mode methods capitalize on the dependence of the mode amplitudes on the source depth. By comparing a set of mode amplitudes extracted from real data to a model, it is possible to determine the source depth. Typically, theoretical mode amplitudes are obtained using propagation equations in a perfect waveguide $[7,8]$ or a normal mode model [17]. Then, the source depth is estimated by matching predicted mode amplitudes to measured mode amplitudes, using a contrast function.

Most classical MMP methods use a vertical line array (VLA) of hydrophones to extract the mode excitation factors. In this case, the recorded signal can be expressed as a linear matrix with one term linked to mode functions at the receiver and one term associated to the source location (which contains mode amplitudes). Then, using the orthogonality of the modes [7], information on source location can be extracted.

It is often more convenient in practice to work using horizontal geometry arrays. For example, sensors placed horizontally along the sea bottom, because they remain stationary, can be left to record continuously for long periods of time. This is not always possible with a vertical array of sensors as vertical arrays are sometimes free floating and move all the time.

This leads to a problem that has not been studied: can we perform MMP using a horizontal line array (HLA) of sensors? The expression of the recorded signal do not lead to a simple extraction of the source information, and mode excitation factors cannot be extracted using the same approach (which is based on the vertical sampling of the data). In this paper, we propose an alternative method, based on the frequency-wavenumber transform $(f-k)$, to achieve mode amplitude extraction using a horizontal line array of sensors.

\section{MATCHED-MODE PROCESSING IN THE FREQUENCY-WAVENUMBER DOMAIN}

\subsection{Motivation and frequency-wavenumber transform}

Let us consider an omnidirectional point source at depth $z=z_{s}$ and range $r=0$ which radiates a broadband signal in a shallow water waveguide. The acoustic field is sampled by a horizontal line array (HLA) of hydrophones placed on the sea bottom (at depth $z=z_{D}$ ). Figure 3 presents the environmental configuration and the source-array geometry.

To perform mode extraction from the recorded signals, frequency-wavenumber domain is used because modes are isolated in this plane. The frequency-wavenumber representation $P_{f k}\left(k_{r}, z_{D}, f\right)$, also called $f-k$ transform, is the $2 \mathrm{D}$ Fourier transform of a section $P\left(r, z_{D}, t\right)$ in time $t$ and distance $r$ at a given depth $z_{D}$. This representation, function of

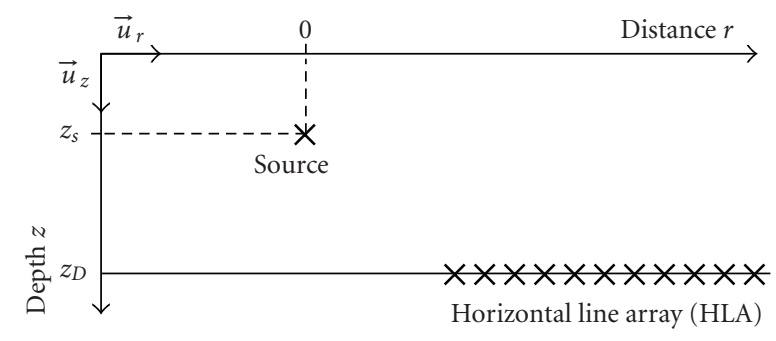

FIgURE 3: Experimental configuration.

the frequency $f$ and wavenumber $k_{r}$, is complex but typically only its modulus is used. The expression of the $f-k$ transform, is then

$$
\begin{aligned}
& P_{f k}\left(k_{r}, z_{D}, f\right) \\
& \quad=\left|\int_{t} \int_{r} P\left(r, z_{D}, t\right) \exp \left(-2 i \pi\left(f t-k_{r} r\right)\right) d t d r\right| .
\end{aligned}
$$

As we use an HLA of sensors, it is possible to build the $f-k$ transform of the recorded data. We consider a white broadband source and use the study of the propagation in a perfect waveguide (Section 2.1). Details of the transformation and hypothesis are given in the Appendix. Then, the theoretical $f-k$ transform of the data recorded at long range, after range normalization, and on an infinite HLA, is

$$
P_{f k}\left(k_{r}, z_{D}, f\right)=\left|B \sum_{m=1}^{+\infty} \psi_{m}\left(z_{s}\right) \psi_{m}\left(z_{D}\right) \delta\left(k_{r}-k_{r m}\right)\right|,
$$

where $B$ is a constant. In the case of a long HLA, the expression (4) remains a valid approximation of the $f-k$ transform. The energy is located on the dispersion curves $\left(k_{r}=k_{r m}\right)$ of the modes. At each frequency, the wavenumber spectrum of the modes is discrete (cf. Section 2.1). The dispersion curves, representing the modes, are separated in the $f-k$ domain [15]. As a result, and using the fact that the HLA is located on the sea bottom (which involves $\left|\psi_{m}\left(z_{D}\right)\right|=1$ ), the $f-k$ transform is

$$
P_{f k}\left(k_{r}, f\right) \approx B \sum_{m=1}^{+\infty}\left|\psi_{m}\left(z_{s}\right)\right| \delta\left(k_{r}-k_{r m}\right) .
$$

Amplitude of the $f-k$ transform along a mode dispersive curve only depends on the mode excitation factor modulus. Using these curves, it will be possible to extract mode excitation factors. Figure 4 shows two examples of $f-k$ representations simulated in a perfect waveguide for two different source depths $z_{s_{1}}=0.2 D$ and $z_{s_{2}}=0.4 D$. For the source at $0.4 D$ (right), mode 3 is not excited, whereas it is for the source located at $0.2 \mathrm{D}$ (left), which is consistent with propagation theory (Figure 2).

In a range-independent Pekeris waveguide (Figure 5) made of a homogeneous fluid layer (velocity $V_{1}$, density $\rho_{1}$, depth $D$ ) overlying a homogeneous fluid half space (velocity $V_{2}$, density $\rho_{2}$ ) with no attenuation, results are almost similar. The main difference is that mode excitation factors are 

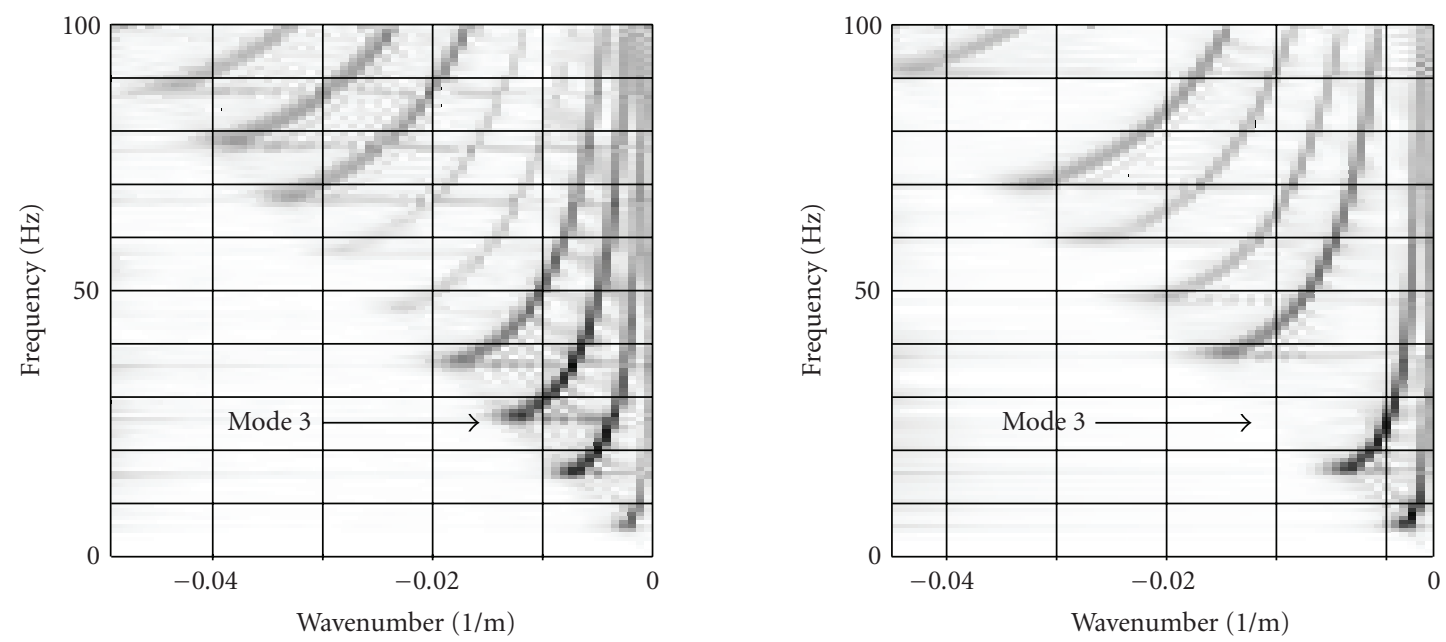

FIGURE 4: $f-k$ representations in a perfect waveguide for a simulated source located at two different depths: $0.2 D$ (left) and $0.4 D$ (right).

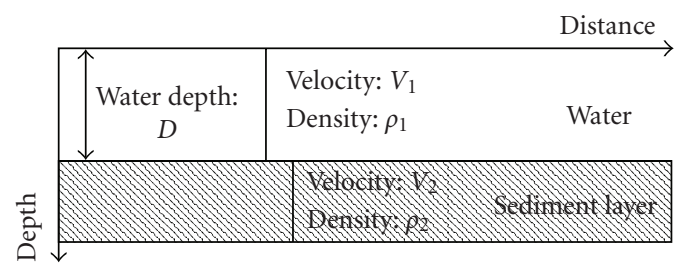

Figure 5: Pekeris waveguide.

a function of the frequency. As a result, the estimated mode amplitude is a mean excitation factor along each dispersion curve. Moreover, mode excitation factors at the bottom interface are not exactly unite and will slightly modify the estimation of the mode amplitudes at the source. These phenomena will not affect the results of the proposed method as the theoretical mode amplitudes will be extracted by the same method.

\subsection{Mode excitation factors and depth estimation}

\subsubsection{Mode excitation factors estimation (or modal filtering)}

The first step of MMP using an HLA consists in extracting mode excitation factors. In geoacoustic inversion, many methods have been developed and applied to extract mode amplitude using a wavenumber transform [10, 12, 13, 18]. In this paper, the extraction is performed by mask filtering in the $f-k$ plane, which allows a simple extraction as long as the environment is known. We can note that if the environment is unknown, geoacoustic parameters can be estimated on the $f-k$ representation [15]. To extract modes, it is necessary to find, in the $f-k$ plane, areas where modes exist. Using propagation theory in a Pekeris waveguide, which is a realistic and simple model for shallow water environments, these areas, called dispersion curves, are defined by

$$
\begin{array}{r}
\tan \left(2 \pi D \sqrt{\frac{f_{m}^{2}}{V_{1}}-k_{r m}^{2}}-\left(m-\frac{1}{2}\right) \pi\right) \\
=\frac{\rho_{1} \sqrt{\left(k_{r m} V_{1} / f_{m}\right)^{2}-\left(V_{1} / V_{2}\right)^{2}}}{\rho_{2} \sqrt{1-\left(k_{r m} V_{1} / f_{m}\right)^{2}}},
\end{array}
$$

where $f_{m}$ is the frequency of the mode $m$ and $k_{r m}$ its horizontal wavenumber. To build masks, we also have to take into account the $V_{1}$ velocity correction (a classical preprocessing in seismic) which modifies $f-k$ representation to provide an $f-k$ representation without spatial aliasing. It consists in applying a time correction along the distance axis $r$. In practice, the recorded signal of each sensor is time shifted so that the direct wave, whose velocity is $V_{1}$, impinges all the sensors at the same time. The consequence of this processing in the $f-k$ plane is that one point $M=\left(f, k_{r}\right)$ is shifted to $M^{\prime}=\left(f, k_{r}-f / V_{1}\right)$.

Figure 6 shows the theoretical $f-k$ representation in a Pekeris waveguide before (left) and after (right) $V_{1}$ velocity correction [15].

After this correction, using (6), and assuming that geoacoustic parameters (velocities, densities, and water depth) are known, we can build a binary mask of the dispersion curve of each mode in the $f-k$ plane. Figure 7 presents this theoretical curve for mode 3 in a Pekeris waveguide defined by $V_{1}=1520 \mathrm{~m} / \mathrm{s}, V_{2}=1875 \mathrm{~m} / \mathrm{s}$, and $D=130 \mathrm{~m}$.

Built masks could be used to extract modes but, in practice, energy of a mode is not located on a line but on a region around this line especially because of the limited length of the array. Consequently, it is necessary to dilate previous theoretical masks. This dilation is also useful because it allows us to take into account environmental mismatch (error on water depth estimation or propagation in a more complex environment than a Pekeris waveguide) which slightly modify the location of dispersion curves. Using this dilation, modes can 

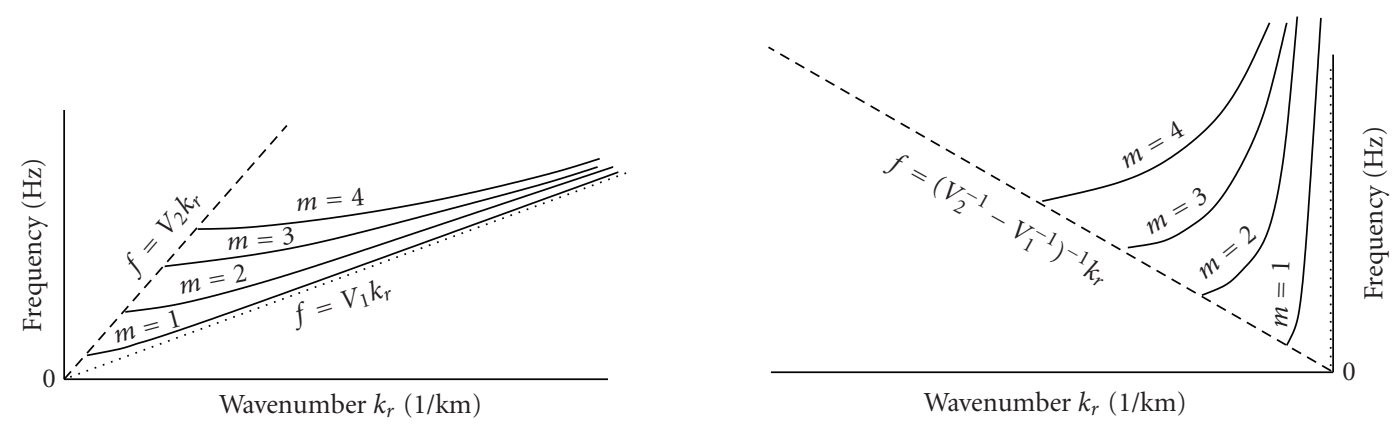

Figure 6: Theoretical $f-k$ representation in a Pekeris waveguide before (left) and after (right) $V_{1}$ velocity correction.

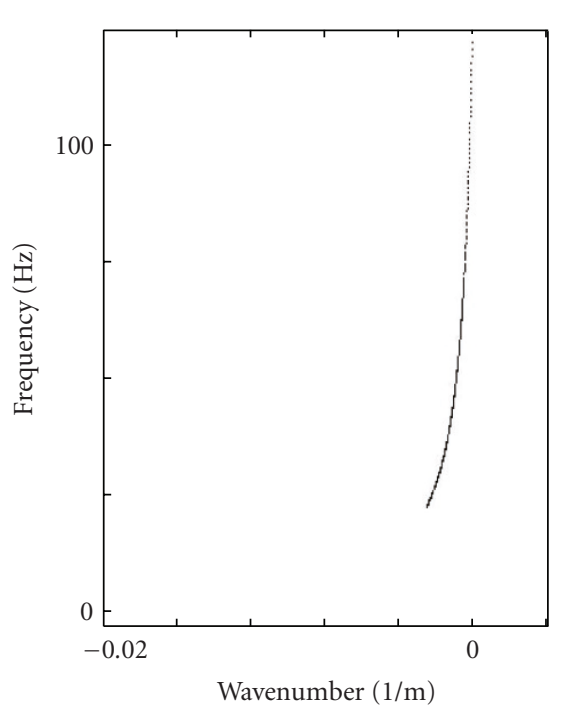

FIgURE 7: Dispersion curve of mode 3 in the $f-k$ domain after $V_{1}$ velocity correction for a Pekeris waveguide: $D=130 \mathrm{~m}, V_{1}=$ $1520 \mathrm{~m} / \mathrm{s}$, and $V_{2}=1875 \mathrm{~m} / \mathrm{s}$.

be extracted even if the environment is not perfectly known. Dilation factor is chosen using $f-k$ representation of the real data, but source depth estimation is not sensitive to it, as long as it is large enough to extract all the mode from the $f-k$ representation and small enough to extract only one mode. After dilation, the binary mask used to extract mode 3 in the Pekeris waveguide presented below is shown in Figure 8.

For each mode, a dilated mask is built and the $f-k$ transform is multiplied by this mask to extract the concerned mode. Figure 9 shows the $f-k$ transform of a real section recorded on an HLA in an oceanic waveguide (which can be modeled by a Pekeris waveguide with $D=130 \mathrm{~m}$, $V_{1}=1520 \mathrm{~m} / \mathrm{s}$, and $V_{2}=1875 \mathrm{~m} / \mathrm{s}$ ). After mask filtering of mode 3 , the filtered $f-k$ representation is plotted in Figure 10.

For each mode $m$, the mean of the $f-k$ representation on the mask gives an estimation of the mean excitation factor modulus $c_{m}$ of this mode. We can note that as we use modulus of the $f-k$ transform, only mode excitation factor modulus is estimated (without its sign), but for the sake

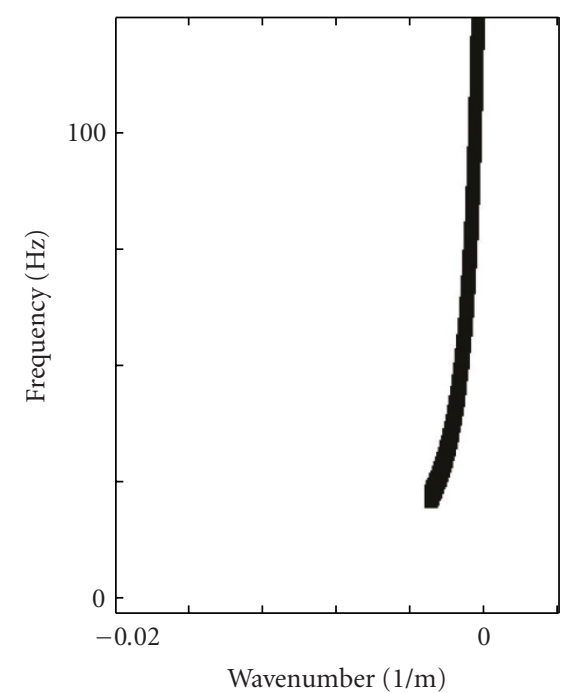

FIGURE 8: Binary mask used to extract mode 3 in the $f-k$ domain for a Pekeris waveguide: $D=130 \mathrm{~m}, V_{1}=1520 \mathrm{~m} / \mathrm{s}$, and $V_{2}=$ $1875 \mathrm{~m} / \mathrm{s}$ after $V_{1}$ velocity correction (black $=1$, white $=0$ ).

of simplicity, it will be called mode excitation factor in the following. Then, to compare real and simulated mode excitation factors, a normalization is made using the closure relationship between modes:

$$
\sum_{m} c_{m}^{2}\left(z_{s}\right)=1
$$

At this step, normalized mode excitation factors $c_{m_{\text {real }}}$ have been extracted from the $f-k$ representation of the real data. We can note that the number of extracted modes is determined by the band of the source signal.

\subsubsection{Depth estimation}

Once mode excitation factors $c_{m_{\text {real }}}$ are extracted for real data, they are used to estimate the source depth. Real mode amplitudes are compared, using a contrast function, to simulated mode amplitudes. To obtain these simulated mode amplitudes, a point source is placed at each depth in the guide. The simulated acoustic field recorded on the HLA is computed. 


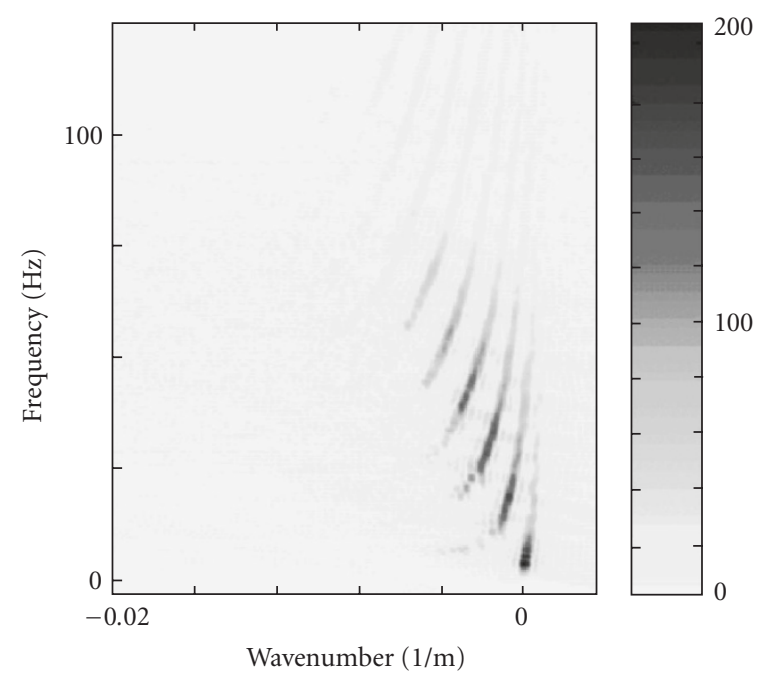

Figure 9: $f-k$ representation of the recorded data.

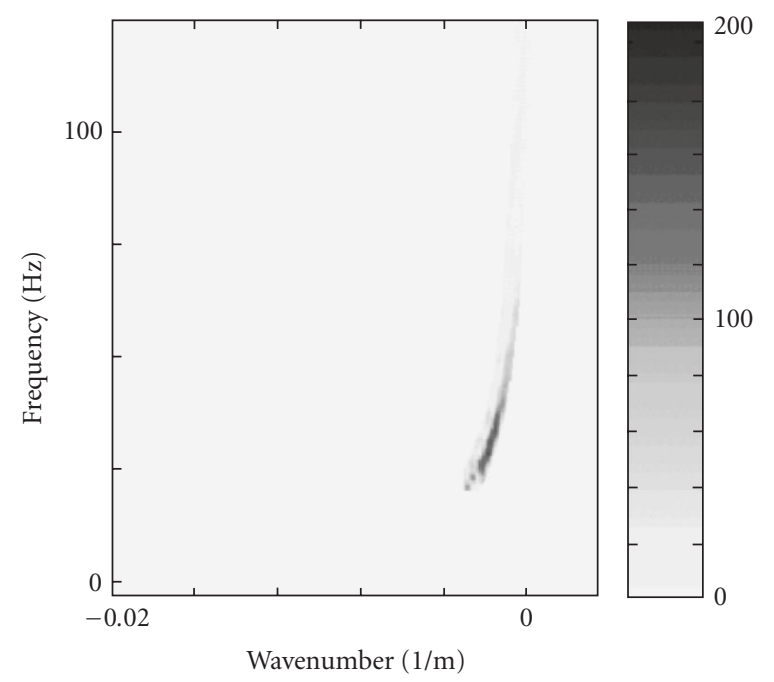

Figure 10: Mode 3 extracted from $f-k$ representation by mask filtering.

Simulated fields are obtained using a finite-difference algorithm, developed by Virieux, which models propagation of $P$ and SV waves in heterogeneous media [19]. Simulations are made in an environment close to the real environment (environment identification is performed using $[15,20]$ ). Then, simulated mode amplitudes $c_{m_{\text {simu }}}$ are extracted using the method presented above (mask filtering).

The last step, to compare measured and simulated mode amplitudes, consists in maximizing the contrast function defined by

$$
G=10 \log _{10}\left(\frac{n_{m}}{\sum_{\text {modes }}\left(c_{m_{\text {simu }}}-c_{m_{\text {real }}}\right)^{2}}\right)
$$

with $n_{m}$ the number of modes. Then the estimated source depth is given by the depth maximizing the contrast function G.

\subsection{Summary of the proposed method and discussion}

To summarize the proposed method, we describe the different steps in the chronological order:

(i) $V_{1}$ velocity correction on the real data: this pre-processing provide an $f-k$ representation without spatial aliasing and involves mode separation in the $f-k$ domain,

(ii) mask building in the $f-k$ plane,

(iii) mode excitation factors estimation on real data $\left(c_{m_{\text {real }}}\right)$,

(iv) simulation of the propagation for different source depths in an environment close to the real environment,

(v) mode excitation factors estimation on simulated data $\left(c_{m_{\text {simu }}}\right)$,

(vi) computation of the contrast function $G$, using real and simulated mode excitation factors.

\section{Discussion}

The main difference between classical MMP and the proposed method is that firstly mode extraction is performed using a horizontal line array. Extraction is based on the frequency-wavenumber transform of the recorded data and on mask filtering. Secondly, another specificity of the method is that masks are built to take into account environmental mismatches, so that the method could be applied on real data for which environment knowledge can be partial. Besides, the presented method is different from classical MMP as theoretical mode amplitudes are extracted from finite difference simulations using the method applied on real data, whereas classical MMP usually use theoretical amplitudes obtained using propagation equations in a perfect waveguide or normal mode models.

\section{SENSITIVITY TO NOISE AND TO ENVIRONMENTAL MISMATCH}

In this section, MMP in the $f-k$ domain is applied on simulated data to estimate sensitivity to noise and robustness against environmental mismatch of the method.

\subsection{Sensitivity to noise}

To study robustness against noise, simulations are made in an oceanic waveguide using a finite-difference algorithm modeling P-SV waves propagation in heterogeneous media [19]. The environment is made of a homogeneous water layer $(D=200 \mathrm{~m})$ overlying a homogeneous solid half space. $\rho$ and $V$ are, respectively, density and sound speed, when two numbers are given for wave velocities, the second number is associated with the respective shear parameter. The source, located in water, has a quasi-white spectrum on the band $0-30 \mathrm{~Hz}$ (Figure 11). The pressure field is recorded on an HLA of 120 sensors placed on the sea bottom and spacing between two hydrophones is $20 \mathrm{~m}$ (this spacing allows us to respect Shannon conditions in space as the wavelength is $50 \mathrm{~m}$ ). Figure 12 presents the source-array geometry and the geoacoustic parameters of the oceanic waveguide. 


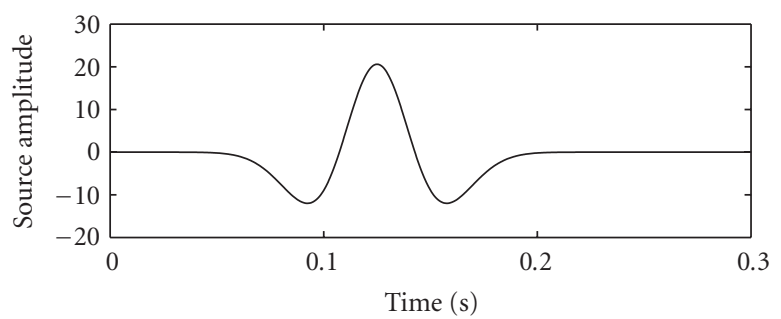

(a)

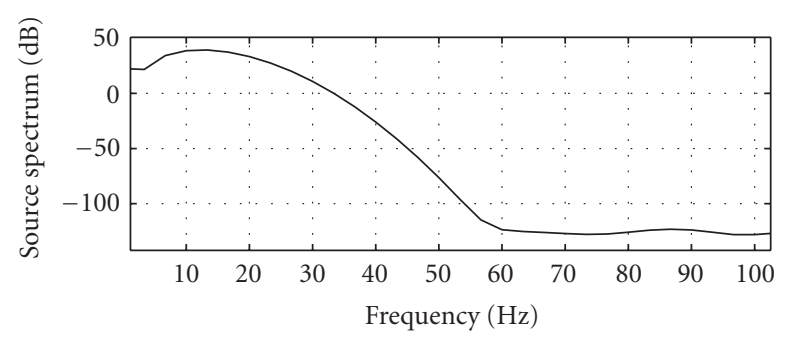

(b)

FIGURE 11: Source signal and its spectrum.

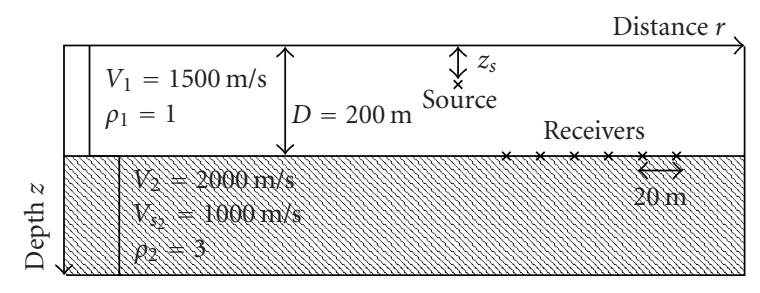

FIGURE 12: Experimental context.

On each simulation, independent Gaussian white noises are added on each sensor. For each signal-to-noise ratio, SNR, $(12 \mathrm{~dB}, 3 \mathrm{~dB}$, and $0 \mathrm{~dB})$, we simulate the propagation of 90 sources located at different depths in the waveguide (every $2 \mathrm{~m}$ from $10 \mathrm{~m}$ to $180 \mathrm{~m}$ ). Source depth is estimated using the method described above and compared to real source depth.

Let us consider the example of a source located at $z_{s}=$ $130 \mathrm{~m}$, the contrast function $G$ for different SNR is plotted in Figure 13. For a high SNR (12 dB-solid line), $G$ presents a maximum for the simulated depth of $130 \mathrm{~m}$. In this case, source depth estimation is perfect $\left(\hat{z}_{s}=130 \mathrm{~m}\right)$ and the difference between the maximum and other local maxima is greater than $23 \mathrm{~dB}$. As SNR decreases, the maximum of the $G$ decreases too, and becomes closer to other local maxima. For an SNR of $0 \mathrm{~dB}$ (dashed line), source depth estimation gives an erroneous result: $\widehat{z}_{s}=98 \mathrm{~m}$. These errors are due to the fact that mode amplitudes are sinusoidal functions: as a result, some depths are "close," in term of mode amplitudes modulus, particularly when the SNR is low (which involves that mode amplitudes are not estimated precisely).

Considering all the simulations, it is possible to study the robustness of the method against noise. Tables 1, 2, and 3 present, respectively, the errors on source depth estimation

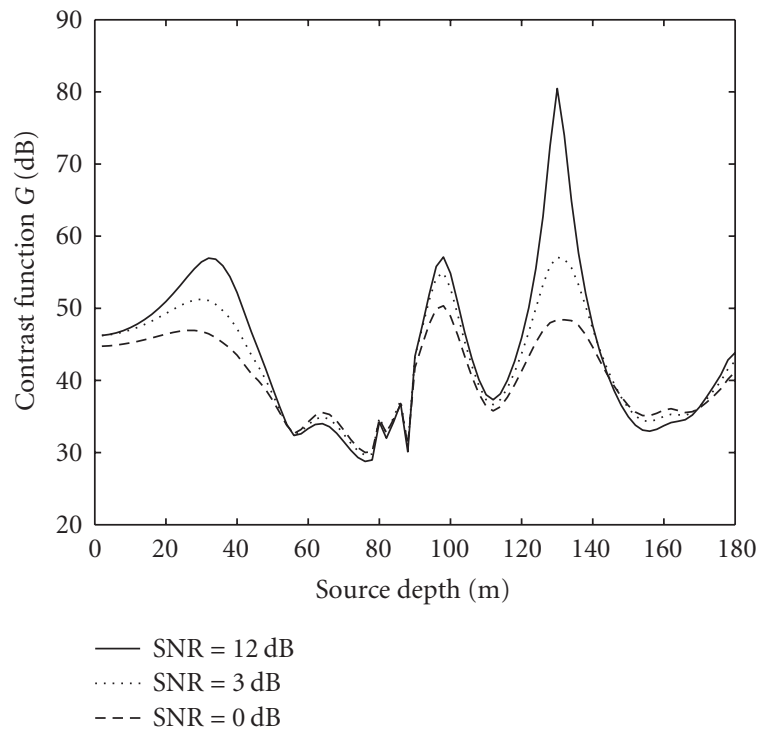

FIGURE 13: Contrast function for a source located at $z_{s}=130 \mathrm{~m}$ for different SNR: $12 \mathrm{~dB}, 3 \mathrm{~dB}$, and $0 \mathrm{~dB}$.

TABLE 1: SNR = $12 \mathrm{~dB}$ : error on source depth estimation.

\begin{tabular}{lc}
\hline \multicolumn{2}{c}{$\mathrm{SNR}=12 \mathrm{~dB}$} \\
\hline Estimation error between & Percentages of estimations \\
\hline $0-2 \mathrm{~m}$ & $98 \%$ \\
$2-4 \mathrm{~m}$ & $2 \%$ \\
+ & $0 \%$ \\
\hline
\end{tabular}

TABLE 2: SNR = $3 \mathrm{~dB}$ : error on source depth estimation.

\begin{tabular}{lc}
\hline \multicolumn{2}{c}{ SNR $=3 \mathrm{~dB}$} \\
\hline Estimation error between & Percentages of estimations \\
\hline $0-2 \mathrm{~m}$ & $61 \%$ \\
$2-4 \mathrm{~m}$ & $12 \%$ \\
$4-6 \mathrm{~m}$ & $6 \%$ \\
$6-10 \mathrm{~m}$ & $7 \%$ \\
+ & $14 \%$ \\
\hline
\end{tabular}

for 3 different SNRs: $12 \mathrm{~dB}, 3 \mathrm{~dB}$, and $0 \mathrm{~dB}$. For a high SNR $(12 \mathrm{~dB})$, source depth is estimated with an error less than $2 \mathrm{~m}$ for all simulated depths. If the SNR decreases to $3 \mathrm{~dB}$, only $61 \%$ of the estimations are made with an error less than $2 \mathrm{~m}$ but there are still 79\% of the estimations with an error smaller than $6 \mathrm{~m}$. When the SNR is low $(0 \mathrm{~dB})$, source depth estimation is not correct ( $43 \%$ of the estimations presents an error greater than $6 \mathrm{~m}$ ) as mode amplitudes cannot be estimated with an acceptable precision.

To conclude about sensitivity to noise, the presented method is quite robust against noise and estimates the source depth with a satisfactory precision as long as the SNR is more than $3 \mathrm{~dB}$. 
TABLE 3: SNR = $0 \mathrm{~dB}$ : error on source depth estimation.

\begin{tabular}{lc}
\hline \multicolumn{2}{c}{ SNR $=0 \mathrm{~dB}$} \\
\hline Estimation error between & Percentages of estimations \\
\hline $0-2 \mathrm{~m}$ & $42 \%$ \\
$2-4 \mathrm{~m}$ & $10 \%$ \\
$4-6 \mathrm{~m}$ & $5 \%$ \\
$6-10 \mathrm{~m}$ & $9 \%$ \\
+ & $34 \%$ \\
\hline
\end{tabular}

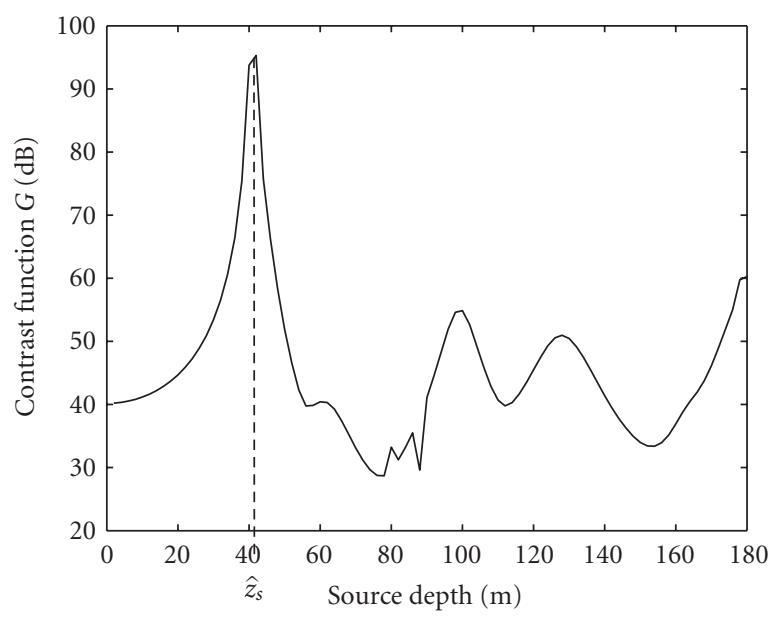

Figure 14: Contrast function $G(\mathrm{~dB})$ for a source located at $z_{s}=$ $40 \mathrm{~m}$ with an error on the water depth of $2 \%$.

\subsection{Sensitivity to environmental mismatches}

Sensitivity to environmental mismatches of MMP methods is a crucial issue and has been studied in the case of a vertical line array $[21,22]$. To study the robustness of the proposed method, we present here the example of a particular environmental mismatch: error on water depth estimation for data recorded on a horizontal line array.

The recorded data are simulated in an oceanic waveguide made of a homogeneous water layer $\left(D=196 \mathrm{~m}, \rho_{1}=1\right.$, $\left.V_{1}=1500 \mathrm{~m} / \mathrm{s}\right)$ and a homogeneous solid half space $\left(\rho_{2}=3\right.$, $\left.V_{2}=2000 \mathrm{~m} / \mathrm{s}, V_{s 2}=1000 \mathrm{~m} / \mathrm{s}\right)$. The experimental configuration is: an underwater source at depth $z_{z}=40 \mathrm{~m}$ and an HLA of 120 sensors placed on the sea bottom (spacing between two hydrophones is $20 \mathrm{~m}$ ).

We suppose that water depth is not exactly known: estimated water depth is $200 \mathrm{~m}$ instead of $196 \mathrm{~m}$. As a result, all the simulations made to extract theoretical mode amplitudes are realized with this estimated water depth. Error between real and estimated water depth has been chosen from mean errors made on environment identification in [15]. Indeed, using the method proposed in $[15,23]$, we note that for a water depth between 100 and $300 \mathrm{~m}$, the error on water depth estimation is less than $2 \%$. To study the robustness of the method to this environmental mismatch, we apply the proposed method and compare real and estimated source depths. Figure 14 presents the contrast function $G$, the

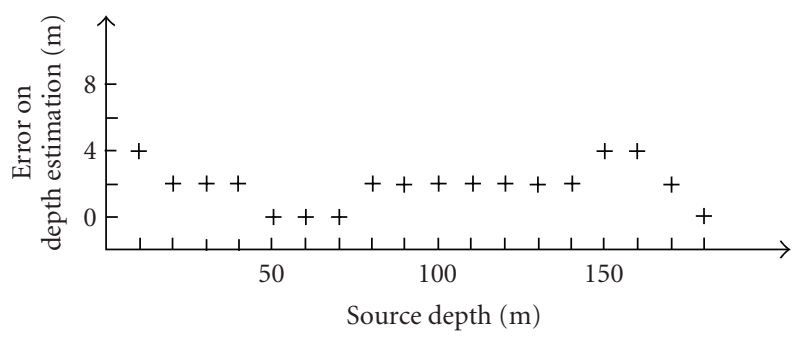

FIGURE 15: Error on source depth estimation with an error on the water depth of $4 \mathrm{~m}$.

estimated source depth is $42 \mathrm{~m}$. Source depth estimation is correct as the error between real and estimated source depths is only $2 \mathrm{~m}$.

Same experiments have been made for other source depths: for each source depth, error on source depth estimation is plotted in Figure 15. The method is robust to this environmental mismatch as error on source depth estimation is less than $4 \mathrm{~m}$.

In this paper, we present in detail robustness of the method against a particular environmental mismatch. Some other works have been made to study robustness against error on water layer velocity or on bottom velocity. For these environmental mismatches, the method provides satisfactory depth estimations: for example, we introduce a gradient velocity (from $1530 \mathrm{~m} / \mathrm{s}$ at the surface to $1500 \mathrm{~m} / \mathrm{s}$ at $40 \mathrm{~m}$ in a $200 \mathrm{~m}$ waveguide), the water depth is estimated assuming that this gradient is unknown. On 10 estimations (source depth between 20 and $160 \mathrm{~m}$ ), the estimation error remains less than $2 \mathrm{~m}$.

As a result, we can note that this method of MMP in the frequency-wavenumber domain is robust to some environmental mismatches (error on water depth, water layer velocity, bottom velocity). This robustness is mainly due to the mask dilation in the $f-k$ domain which allows us to take into account differences between real and simulated dispersive curves.

\section{APPLICATION ON REAL DATA}

After robustness against noise and environmental mismatches studies, we apply the method described in Section 3 on two sets of real data. The first set has been recorded in the North Sea in an environment close to a Pekeris waveguide. On these data, source depth is estimated but it is not possible to estimate the estimation error as source depth was not precisely known during the survey.

For the second set, we performed ultrasonic experiments in a perfectly known environment: a waveguide made of a layer of water overlying a layer of steel. In this case, we apply the presented method and compare estimated and real source depths.

\subsection{North Sea data}

The experimental geometry is shown in Figure 16. The source is an airgun moving from one location to another, 


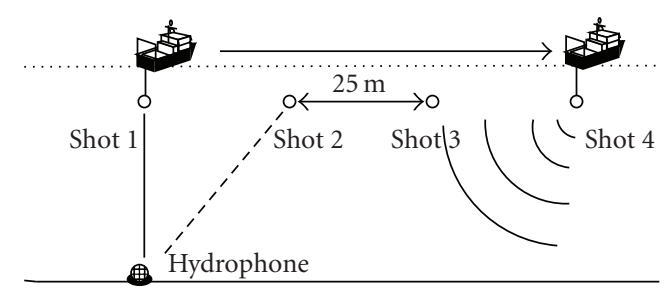

FIGURE 16: North Sea data: source-array geometry.

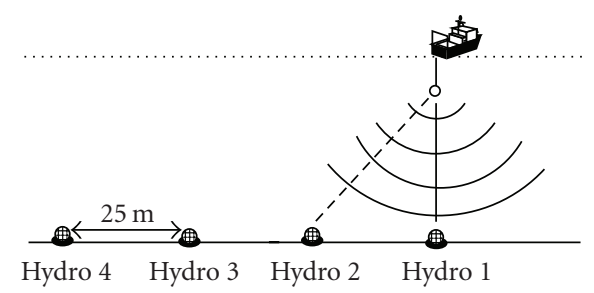

FIGURE 17: North Sea data: equivalent geometry.

making one shot every $25 \mathrm{~m}$. The receiver is a hydrophone placed on the sea bottom. As environment is range independent, this geometry creates synthetic aperture and is equivalent to that presented in Figure 17. Pressure field is recorded on a synthetic antenna of 240 hydrophones, which will allows us to use the method described above. Initial data are time corrected with velocity $V_{1}=1520 \mathrm{~m} / \mathrm{s}$, results in the timedistance domain are plotted in Figure 18. On this figure, we can see different waves (reflected, refracted) but modes cannot be isolated. Then, data recorded between 2 and $6 \mathrm{~km}$ in range are used to compute the frequency-wavenumber transform (Figure 19) and 7 modes can be identified on the $f-k$ transform.

Then, binary masks in the $f-k$ plane are built using Pekeris theory (Figure 20) and geoacoustic parameters are estimated by [15] $V_{1}=1520 \mathrm{~m} / \mathrm{s}, V_{2}=1875 \mathrm{~m} / \mathrm{s}$, and $D=130 \mathrm{~m} .7$ modes are extracted from the $f-k$ representation of the real data (Figure 21 shows extraction of modes $1,2,3$, and 4), and mode excitation factors are estimated (Figure 22-solid line).

Simulations are made in an environment similar to the real environment (identification is made using [15]) and mode excitation factors are extracted on each simulation: Figure 22 shows examples of mode amplitudes for two different simulated source depths: $z_{s}=19 \mathrm{~m}$ and $z_{s}=70 \mathrm{~m}$. For the source located at $z_{s}=70 \mathrm{~m}$, real and simulated mode amplitudes are very different whereas they are close for the simulated source located at $z_{s}=19 \mathrm{~m}$. Then, to estimate the source depth, the contrast function $G$, which compares real and simulated mode amplitudes is computed (Figure 23) and its maximum gives us an estimated source depth of

$$
\hat{z}_{s}=19 \mathrm{~m} .
$$

We do not have the exact value of the source depth but as the source was an airgun, its depth was between 10 and $20 \mathrm{~m}$ which is consistent with the estimated source depth.

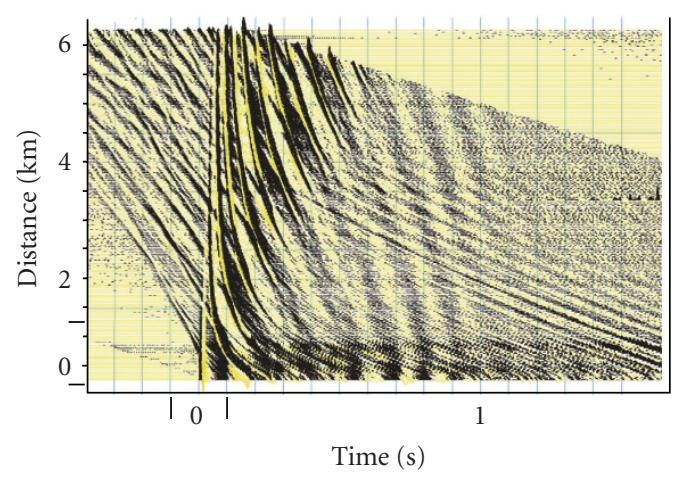

FIGURE 18: North Sea data: time-distance representation of the recorded data after $V_{1}$ velocity correction.

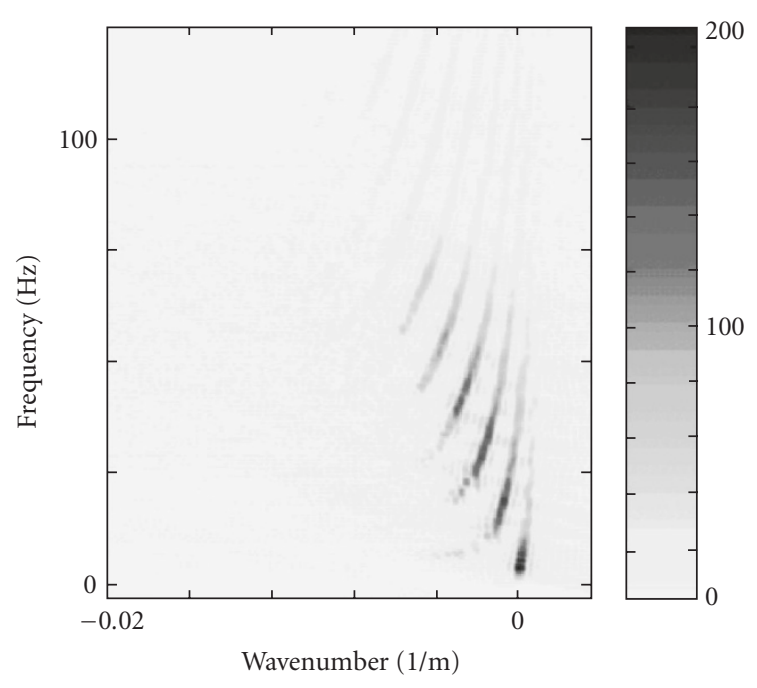

FIgURE 19: North Sea data: frequency-wavenumber representation.

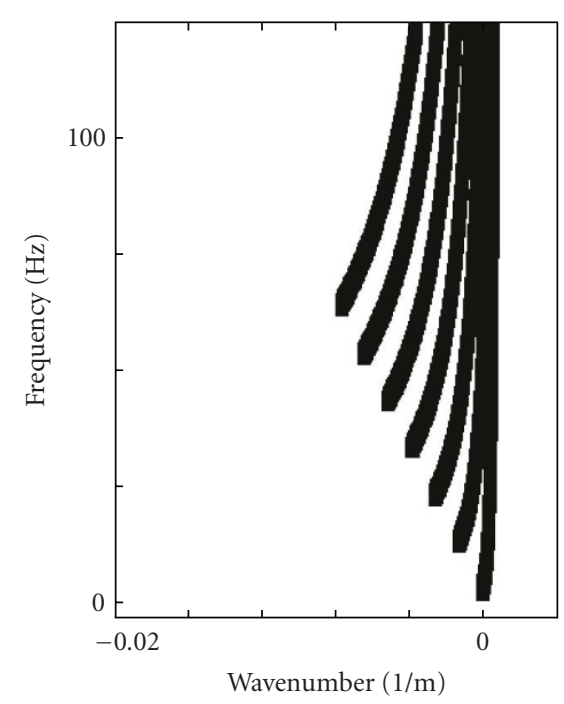

FIgURE 20: North Sea data: masks of the modes in the $f-k$ plane. 


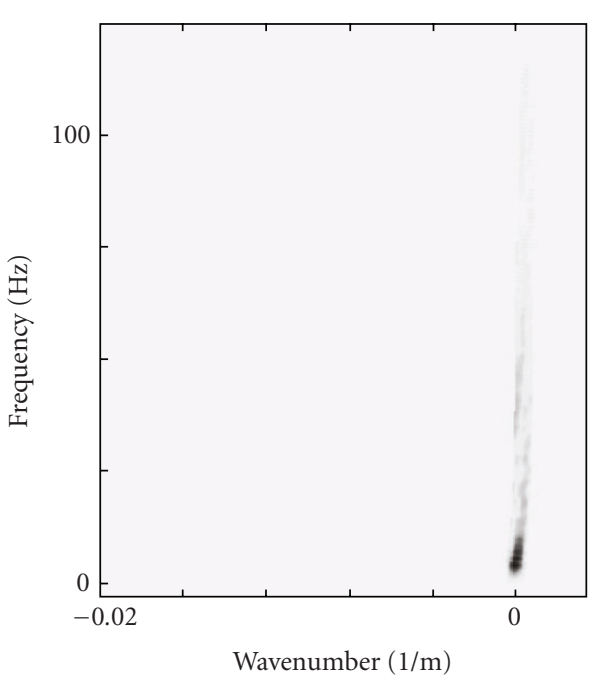

(a)

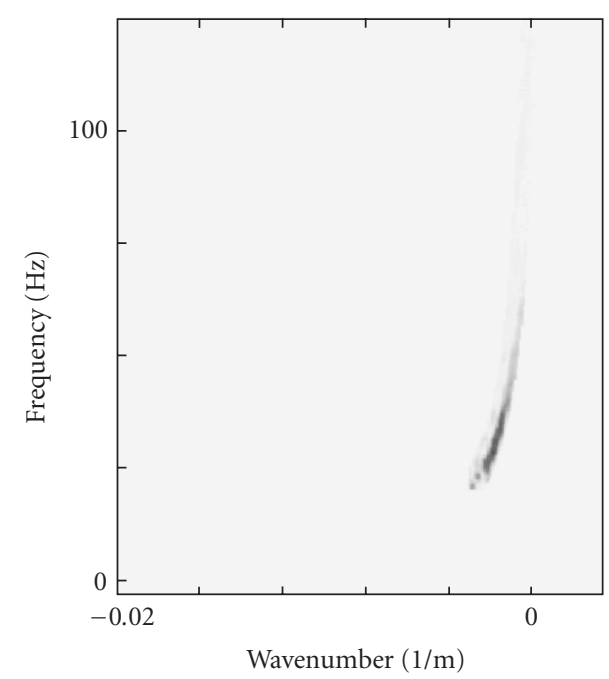

(c)
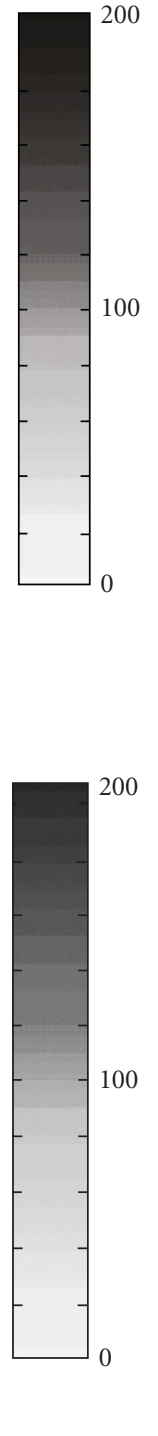

Figure 21: North Sea data: modes 1, 2, 3, and 4 extracted from real data in the $f-k$ plane; (a) mode 1, (b) mode 2, (c) mode 3, and (d) mode 4 .

\subsection{Ultrasonic experiments}

As we want to estimate error on source depth estimation, the proposed method is applied on ultrasonic data recorded in a perfectly known environment and source-array geometry. Experiments have been performed at the Marine Physical Laboratory (SCRIPPS Institution of Oceanography, La Jolla), in collaboration with Dr. P. Roux.

Ultrasonic experiments in tanks are often used in underwater acoustics as they emulate shallow water waveguides: indeed, by multiplying the frequency by a factor $x$, distances are divided by the same factor. As acoustic and elastic propagation properties are not affected by this scaling down, it is possible to achieve "oceanic experiments" in a simple tank. In this section, we will first present the experimental context. Then source depth will be estimated on many recorded data

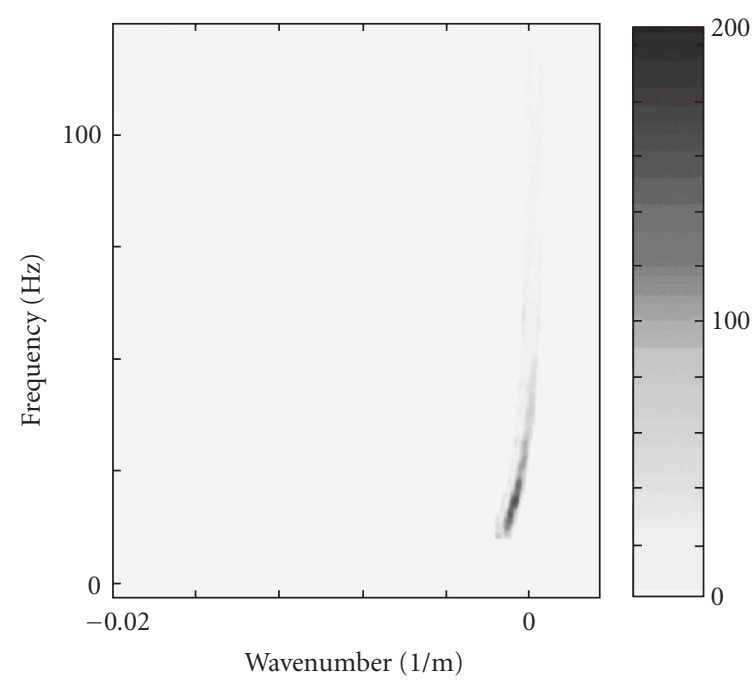

(b)

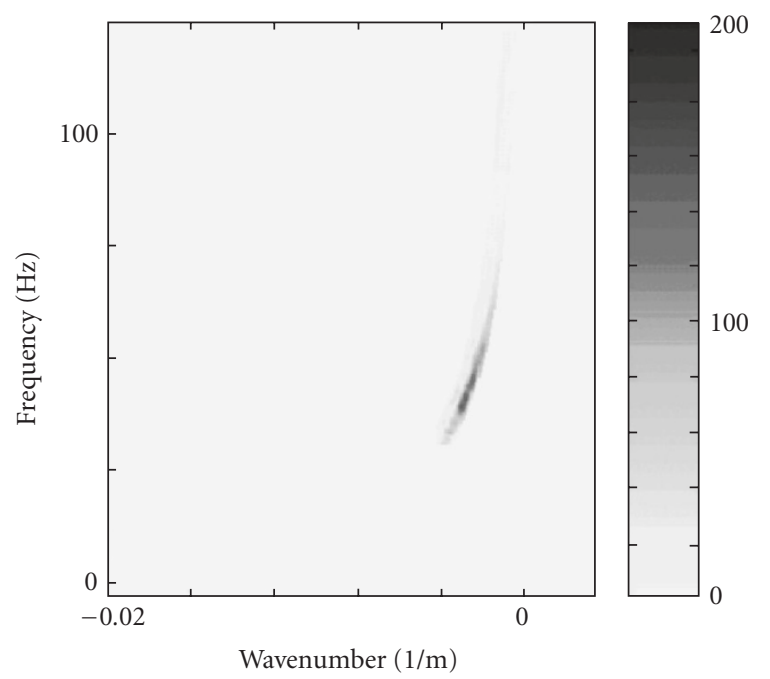

(d) 


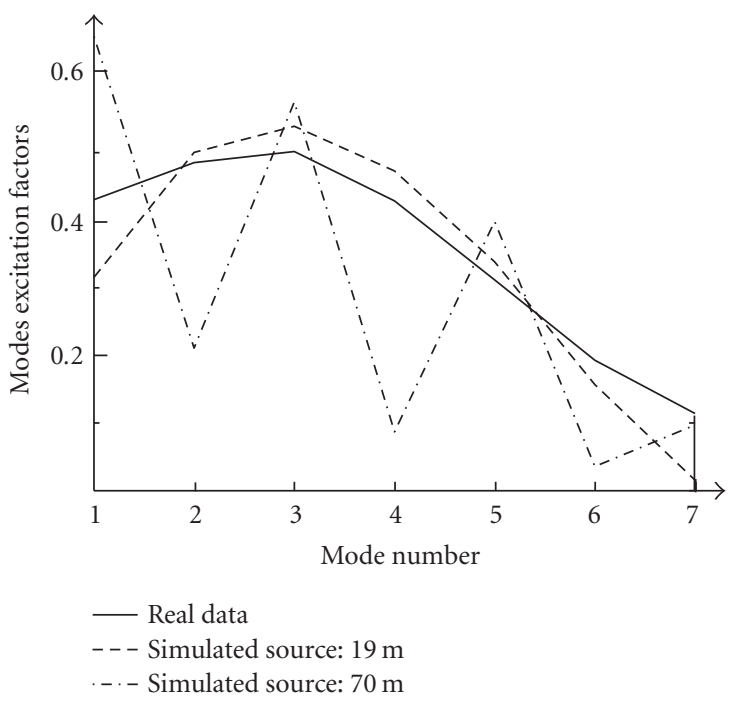

Figure 22: North Sea data: mode excitation factors extracted from real data and from two simulated data at $z_{s}=19 \mathrm{~m}$ and $z_{s}=70 \mathrm{~m}$.

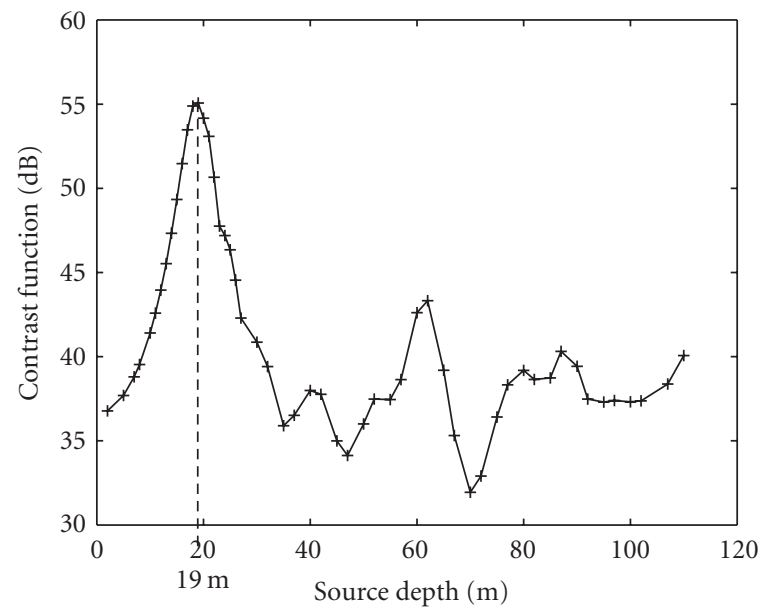

FIgURE 23: North Sea data: contrast function $G(\mathrm{~dB})$ as a function of the simulated source depth.

result, modal filtering method in the frequency-wavenumber plane can be applied to the recorded data if we take into account this property.

As the length of the tank is $1 \mathrm{~m}$, we choose a scaling factor of $10^{4}$ to simulate an oceanic propagation of $10 \mathrm{~km}$. In the following, we will talk about "reduced scales" for distances in the tank and "oceanic scales" for equivalent distances in an oceanic environment. The water depth is $266 \mathrm{~m}$ in oceanic scale.

\section{The source}

A point-like ultrasonic source is located on one side of the waveguide, in the water layer. We want to emit a white signal in the band $100-500 \mathrm{kHz}$ in reduced scale $(10-50 \mathrm{~Hz}$ in oceanic scale). Considering the band of the transducer, it is

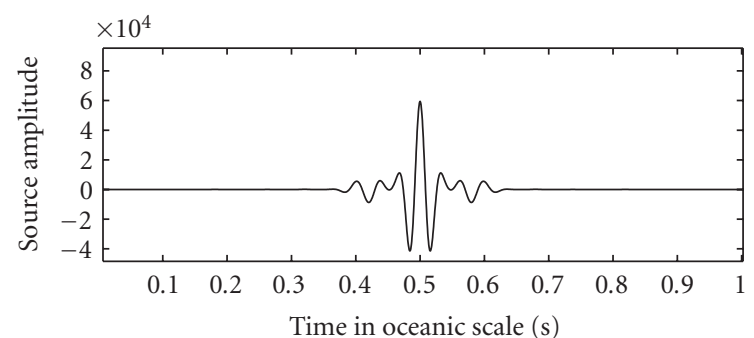

(a)

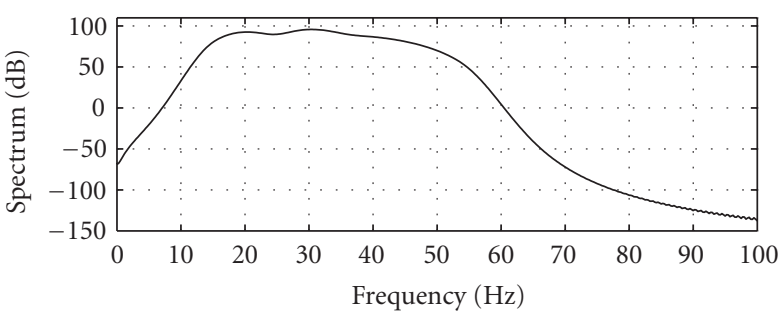

(b)

FIGURE 24: Ultrasonic experiments: equivalent source signal (a) and energy spectrum (b).

TABLE 4: Ultrasonic experiments: source-array geometry.

\begin{tabular}{lcc}
\hline & In reduced scale & In oceanic scale \\
\hline Spacing between receivers & $1 \mathrm{~mm}$ & $10 \mathrm{~m}$ \\
Distance source first receiver & $400 \mathrm{~mm}$ & $4 \mathrm{~km}$ \\
Distance source last receiver & $913 \mathrm{~mm}$ & $9,13 \mathrm{~km}$ \\
\hline
\end{tabular}

necessary to use three different source signals. Each signal is a chirp, as it permits to emit a powerful signal, in the band:

(i) band 1: $100-300 \mathrm{kHz}$,

(ii) band 2: $250-350 \mathrm{kHz}$,

(iii) band 3: $300-500 \mathrm{kHz}$.

The drawback of using a chirp is its duration. To solve this problem, we realize the cross-correlation between each recorded signal and the source signal. Indeed, this is equivalent to have emitted the auto-correlation of the source signal, which is shorter in time. Besides, as the guide is time invariant, the emission of the three signals is equivalent to the emission of their sum. As a result, the equivalent emitted signal is the sum of the three chirp auto-correlations (Figure 24), which is white in the band $100-500 \mathrm{kHz}$ in reduced scale (10-50 $\mathrm{Hz}$ in oceanic scale).

The source is mobile along the depth axis $z$ and several recordings are made for different source depths.

\section{The receiver}

The receiver is a hydrophone placed on the steel which moves horizontally to create a synthetic antenna. The antenna is made of 514 receivers and begins at a horizontal distance of 


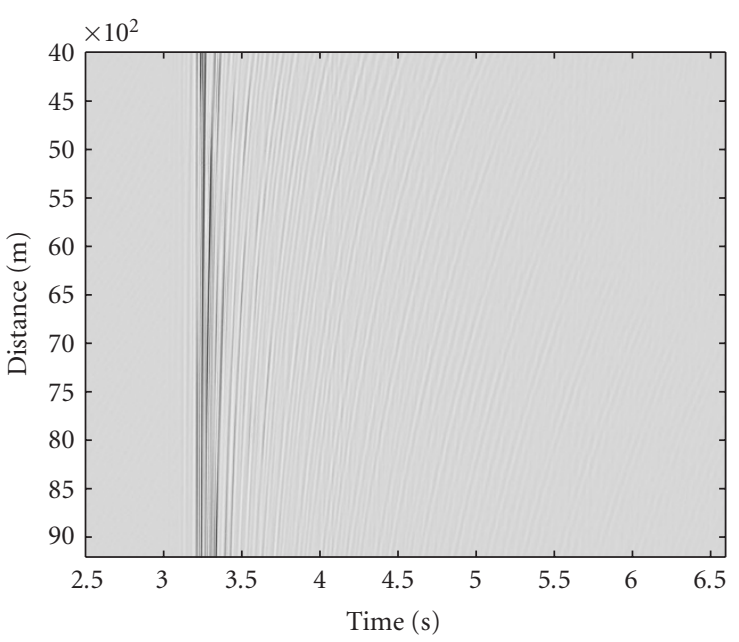

FIgURE 25: Ultrasonic experiments: recorded section after $V_{1}$ velocity correction for a source located at $z_{s}=70 \mathrm{~m}$.

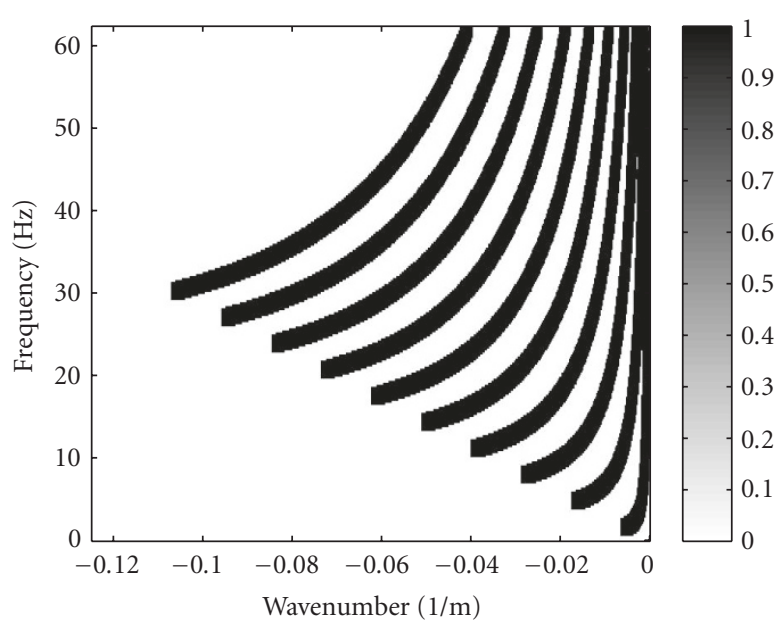

FIGURE 26: Ultrasonic experiments: binary masks of the modes in the $f-k$ plane.

$400 \mathrm{~mm}$ in reduced scale ( $4 \mathrm{~km}$ in oceanic scale) from the source. Spacing between two elements of the HLA is $1 \mathrm{~mm}$ in reduced scale ( $10 \mathrm{~m}$ in oceanic scale). Table 4 summarizes the source-array geometry in reduced and oceanic scales.

For the sake of simplicity, quantities will be given in oceanic scale in the following. As the source is moving, it is possible to record several sets of data. 22 experiments are made for different source depths $z_{s}$ : from $30 \mathrm{~m}$ to $240 \mathrm{~m}$. We first present the example of a source located at $z_{s}=70 \mathrm{~m}$ and then study results obtained for other source depths.

\subsubsection{Example: source located at $z_{s}=70 \mathrm{~m}$}

(a) The first step consists in applying a $V_{1}$ velocity correction to the recorded data. The water layer velocity is known $V_{1}=$ $1487 \mathrm{~m} / \mathrm{s}$, and the recorded section, after velocity correction, is shown in Figure 25.

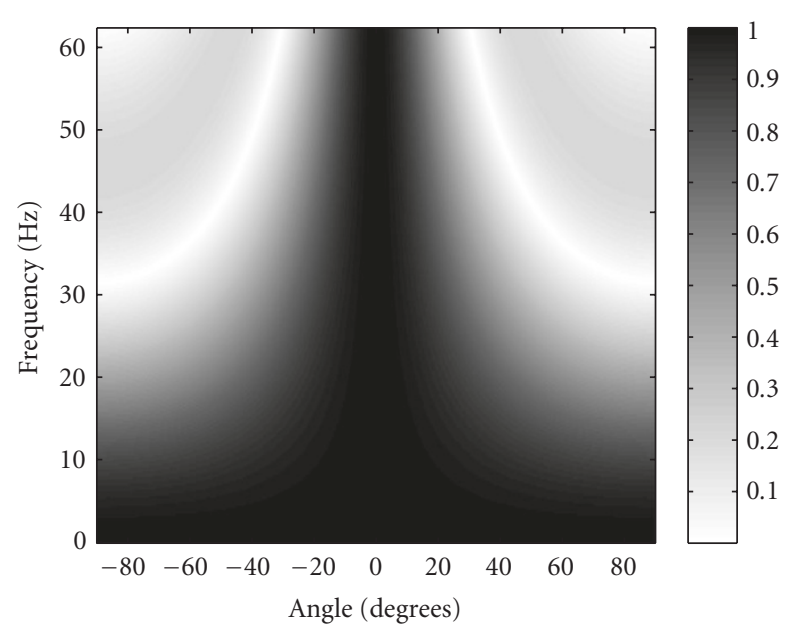

FIGURE 27: Ultrasonic experiments: source directivity as a function of incident angle and frequency.

(b) Then, binary masks are built in the $f-k$ plane. As mentioned above, the ultrasonic waveguide, made of water and steel, is close to a Pekeris waveguide with steel shear-wave velocity (which is $2780 \mathrm{~m} / \mathrm{s}$ ) for bottom layer velocity. Thus, it is still possible to build masks in the $f-k$ plane using $V_{1}=1487 \mathrm{~m} / \mathrm{s}, V_{2}=2780 \mathrm{~m} / \mathrm{s}$, and $D=266 \mathrm{~m}$ (Figure 26).

(c) Binary masks are used to extract mode amplitudes on real data. As the previous study was made for an omnidirectional source, which is not the case here, it is necessary to correct source directivity effect before mask filtering.

The source can be considered as an infinite line in the perpendicular direction to the plane of propagation and has a size $a=15 \mathrm{~m}$ in the direction $z$. Its directivity function $F(\theta, f)$, plotted in Figure 27, can be expressed as a function of incident angle $\theta$ and frequency $f$ by

$$
F(\theta, f)=\left|\sin c\left[\frac{\pi a f}{V_{1}} \sin \theta\right]\right|
$$

Thus, applying the function $1 / F(\theta, f)$, we compensate source directivity. This function is computed only for points where modes exist, that is, on masks in the $f-k$ plane. On these regions the directivity function $F(\theta, f)$ is never equal to zero. $f-k$ transform of the recorded data after source directivity correction is shown in Figure 28. On this representation, we extract mode amplitudes by mask filtering.

(d) Then, simulations are made in an environment close to the real environment. Geoacoustic parameters are estimated using $[15,24]$. As the source height is $15 \mathrm{~m}$, we compute simulations for sources between 10 and $240 \mathrm{~m}$ every $10 \mathrm{~m}$, and on each simulation, mode excitation factors are extracted by mask filtering.

(e) Finally, the contrast function $G$ is computed (Figure 29) and source depth is estimated:

$$
\hat{z}_{s}=70 \mathrm{~m}
$$




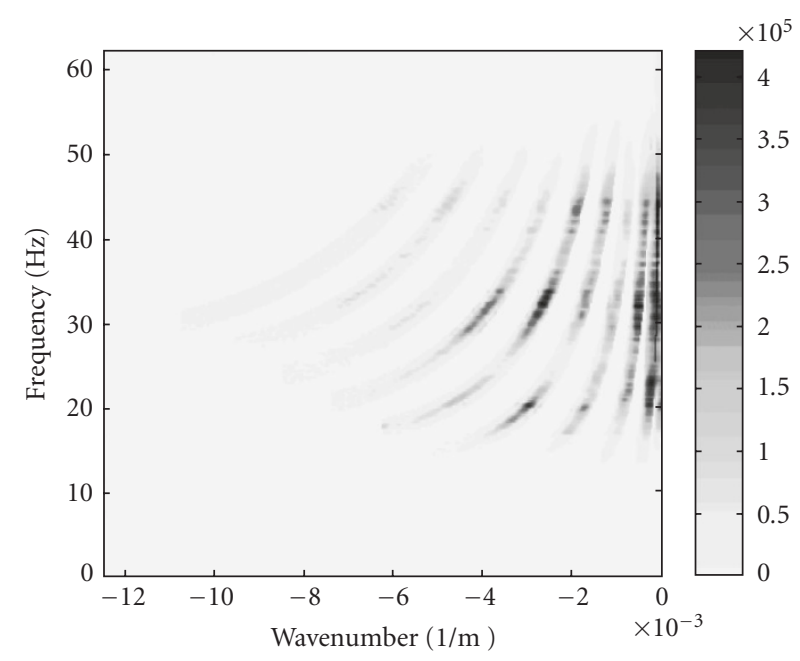

FIgURE 28: Ultrasonic experiments: $f-k$ transform after source directivity correction.

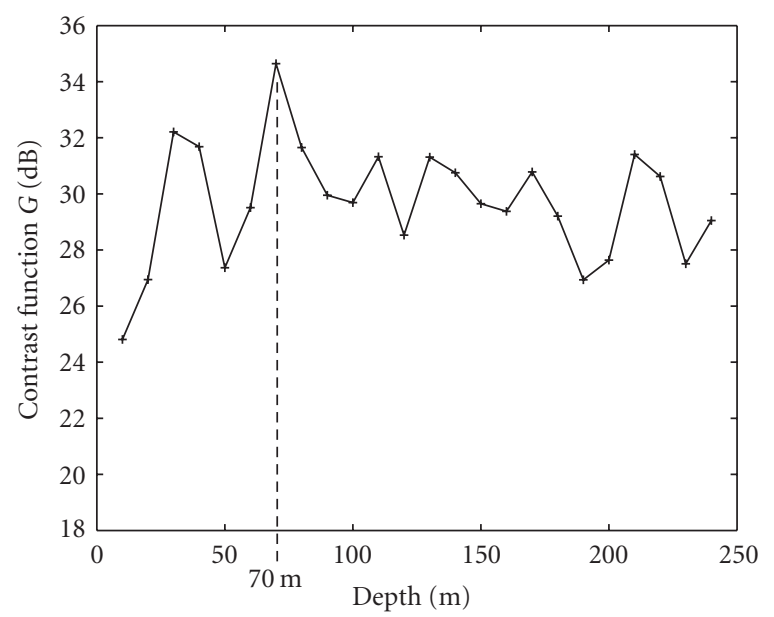

FIGURE 29: Ultrasonic experiments: contrast function $G$ for a source located at $z_{s}=70 \mathrm{~m}$.

\subsubsection{Results analysis}

As environment and source location were perfectly known, it is possible to study error on source depth estimation on the different experiments. Considering source height, source depth estimation is correct if estimation error is less than or equal to $10 \mathrm{~m}$. Table 5 shows results obtained for different source depths: the method gives satisfactory results for 15 source depths on 22 . These results have been studied in detail in [23] to explain estimation errors.

To improve source depth estimation, we propose to use mode excitation factors with their sign (instead of mode excitation factors modulus). Indeed, some source depths can be "close" in term of mode amplitudes modulus and using the sign of these coefficients will permit to separate these source depths.
TABLE 5: Ultrasonic experiments: error on source depth estimation for the 22 experiments without using sign of mode amplitudes.

\begin{tabular}{lc}
\hline Estimation error & Source depth \\
\hline Inferior to $10 \mathrm{~m}$ & $40,70,80,90,100,110,140,210$, and $240 \mathrm{~m}$ \\
Equal to $10 \mathrm{~m}$ & $30,50,60,120,200$, and $220 \mathrm{~m}$ \\
Superior to $10 \mathrm{~m}$ & $130,150,160,170,180,190$, and $230 \mathrm{~m}$ \\
\hline
\end{tabular}

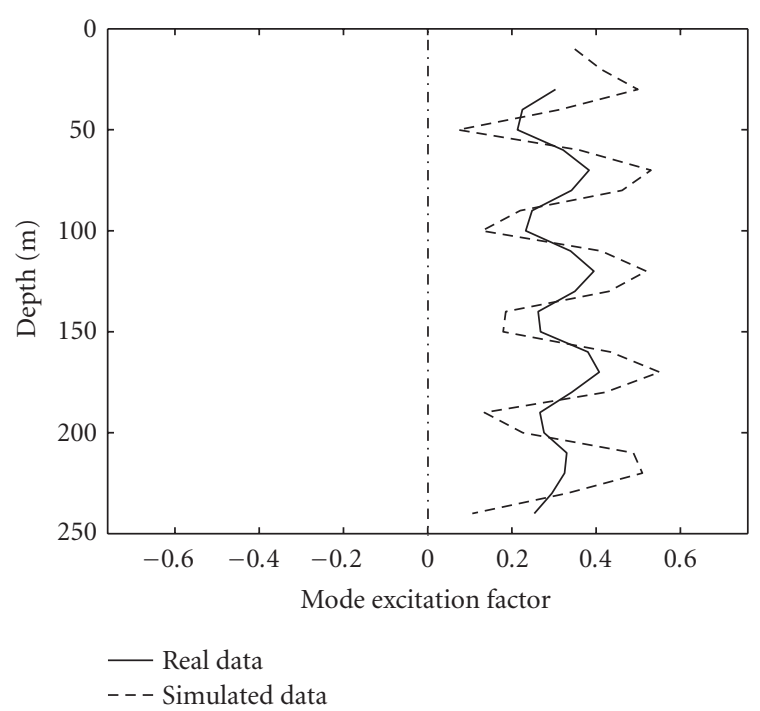

FIGURE 30: Ultrasonic experiments: mode 6 amplitude modulus for real and simulated data.

\subsubsection{Sign of mode excitation factors}

Mode amplitudes with their signs carry more information than their modulus, but sign of these coefficients cannot be easily extracted from $f-k$ representation. In our case, it is possible to extract sign of mode amplitudes as we recorded signals for many source depths in the guide. This extraction is made [23], and we study the influence of this sign of source depth estimation. Figures 30 and 31 show, respectively, mode 6 amplitude modulus and mode 6 amplitude with sign, for real and simulated data and for all source depths.

Using the sign of mode amplitudes is useful as they are sinusoidal functions: as a result, the "frequency" of these functions are divided by two when the sign is known and there are fewer depths that are "close" to each other. The consequence on contrast function is that the maximum is further from other local maxima: Figure 32 shows contrast functions obtained for a source located at $z_{s}=70 \mathrm{~m}$ using mode amplitudes modulus and mode amplitudes with their signs. As a result, source depth estimation is improved using the sign of mode amplitudes.

Results obtained using mode amplitudes with their signs are shown in Table 6 for all the sets of data. In this case, 21 source depth estimations on 22 are correct, which constitutes a great improvement of the method.

At this step, we can note that mode amplitude sign is an important information for efficient source depth estimation. Methods estimating this sign, on $f-k$ phase for example, 


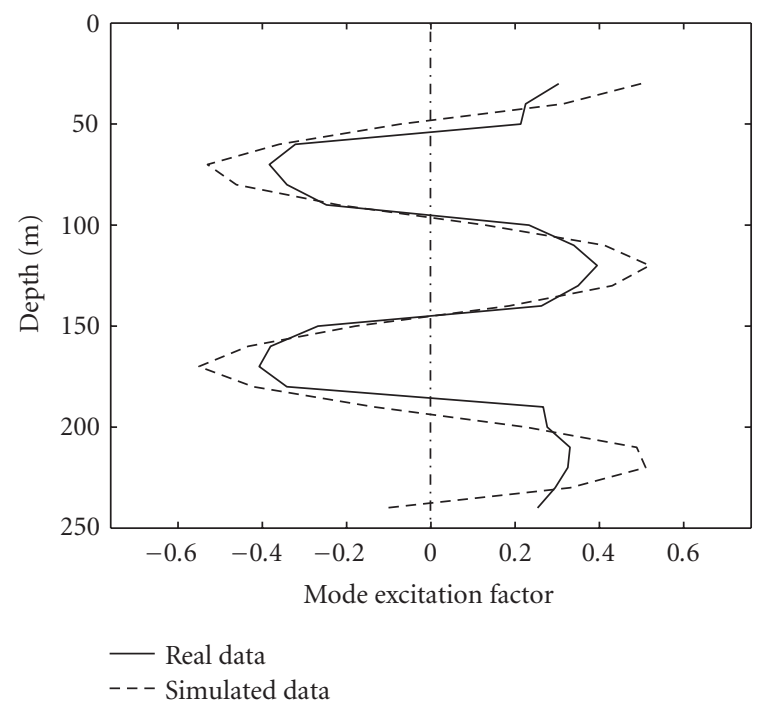

FIGURE 31: Ultrasonic experiments: mode 6 amplitude for real and simulated data.

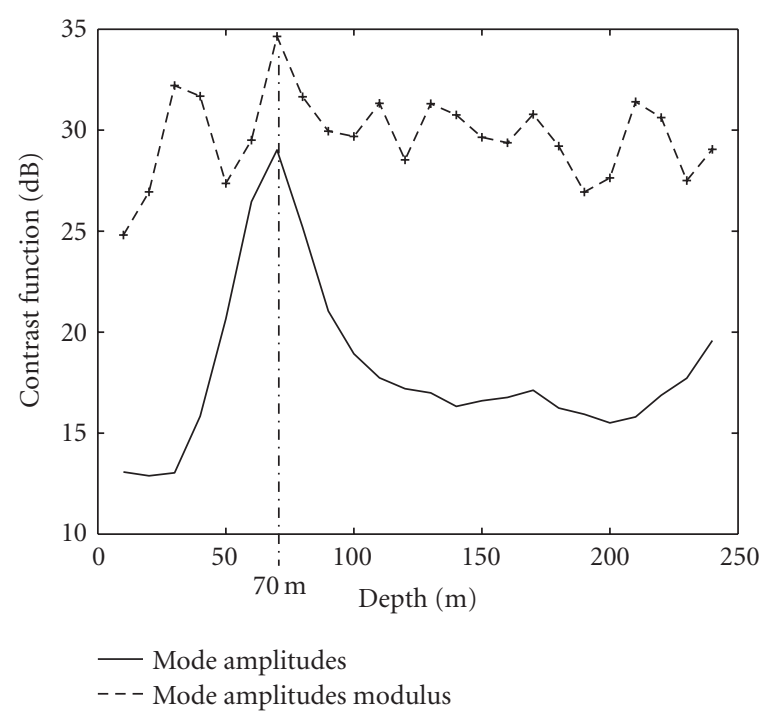

FIGURE 32: Ultrasonic experiments: contrast function $G$ for a source located at $z_{s}=70 \mathrm{~m}$ using sign of mode amplitudes and using mode amplitudes modulus.

must be developed. In this case, localization by MMP using an HLA is drastically improved and gives satisfactory results.

\section{CONCLUSION}

Matched-mode processing methods are widely used to localize underwater sources. These methods, developed for underwater sources in shallow water environments, typically used a vertical line array of sensors to achieve mode amplitudes extraction and source localization. In the case of a horizontal line array, which can be more appropriate for practical applications, these methods cannot be applied.
TABLE 6: Ultrasonic experiments: error on source depth estimation for the 22 experiments using sign of mode amplitudes.

\begin{tabular}{lc}
\hline Estimation error & Source depth \\
\hline Inferior to $10 \mathrm{~m}$ & $30,60,70,80,90,100,110,120,130,140$ \\
& $150,160,170,200,210$, and $240 \mathrm{~m}$ \\
Equal to $10 \mathrm{~m}$ & $40,50,180,190$, and $220 \mathrm{~m}$ \\
Superior to $10 \mathrm{~m}$ & $230 \mathrm{~m}$ \\
\hline
\end{tabular}

In this paper, we propose an original and simple method, based on the frequency-wavenumber transform to extract mode amplitudes and achieve matched-mode processing to estimate source depth. This method has been described and its robustness against noise and some environmental mismatches (error on water depth, water layer velocity, bottom velocity) has been studied. Then, we validated it on several real data. The first set of data, recorded in the North Sea, allows us to show that MMP in the frequency-wavenumber plane gives satisfactory results in a real environment close to a Pekeris waveguide. The second set of data are ultrasonic data, recorded in a tank. For this one, we estimate error on source depth estimation as the environment was perfectly known. Moreover, we show on these data that the use of mode amplitudes sign can improve source depth estimation. A method to extract this sign on at sea data (for which we do not possess sources at many different depths) should be developed in the future to improve source depth estimation.

\section{APPENDIX}

\section{A. FREQUENCY-WAVENUMBER TRANSFORM IN A PERFECT WAVEGUIDE}

Let us consider a monochromatic source (with angular frequency $\omega=2 \pi f$ ) at depth $z_{s}$ in a perfect waveguide. Pressure recorded at $M(r, z)$ can be expressed, at long range, by

$$
P(r, z, t)=A \sum_{m=1}^{+\infty} \psi_{m}\left(z_{s}\right) \psi_{m}(z) \frac{\exp \left(-2 i \pi k_{r m} r\right)}{\sqrt{k_{r m} r}} \exp (2 i \pi f t)
$$

where $A$ is a constant. For a broadband source (with spectrum $S(f)$ ), this expression becomes

$$
\begin{aligned}
P(r, z, t)= & A \sum_{m=1}^{+\infty} \psi_{m}\left(z_{s}\right) \psi_{m}(z) \\
& \times \int_{\nu} \frac{\exp \left(-2 i \pi k_{r m} r\right)}{\sqrt{k_{r m} r}} S(\nu) \exp (2 i \pi v t) d v
\end{aligned}
$$

where $k_{r m}=k_{r m}(v)$. Then, frequency-wavenumber transform of a section recorded on an HLA placed on the sea bottom 
(at depth $z_{D}$ ) can be expressed by

$$
\begin{aligned}
& P_{f k}\left(k_{r}, z_{D}, f\right) \\
&=\left|\int_{r} \int_{t} P\left(r, z_{D}, t\right) \exp \left(-2 \pi i\left(f t-k_{r} r\right)\right) d t d r\right| \\
&= \mid A \sum_{m=1}^{+\infty} \psi_{m}\left(z_{s}\right) \psi_{m}\left(z_{D}\right) \\
& \times \int_{r} \int_{\nu} \int_{t} \frac{\exp \left(-2 i \pi k_{r m} r\right)}{\sqrt{k_{r m} r}} S(\nu) \exp (2 i \pi v t) \\
& * \exp (-2 i \pi f t) \exp \left(2 i \pi k_{r} r\right) d t d v d r \mid
\end{aligned}
$$

Then, taking into account normalization performed on the data along the distance $r$, we obtain

$$
\begin{aligned}
& P_{f k}\left(k_{r}, z_{D}, f\right) \\
& =\mid A \sum_{m=1}^{+\infty} \psi_{m}\left(z_{s}\right) \psi_{m}\left(z_{D}\right) \\
& \quad \times \int_{r} \int_{\nu} \int_{t} \exp (2 i \pi v t) \exp (-2 i \pi f t) \\
& \quad * S(\nu) \exp \left(-2 i \pi\left(k_{r m}-k_{r}\right) r\right) d t d \nu d r \mid .
\end{aligned}
$$

Integration over $t$ and $r$, using the definition of the delta function yields

$$
\begin{aligned}
& P_{f k}\left(k_{r}, z_{D}, f\right) \\
& \quad=\left|A \sum_{m=1}^{+\infty} \psi_{m}\left(z_{s}\right) \psi_{m}\left(z_{D}\right) S(f) \int_{r} \exp \left(-2 i \pi\left(k_{r m}-k_{r}\right) r\right) d r\right| .
\end{aligned}
$$

At this step, we have to study the antenna length. If we consider a finite HLA, then expression of the integral is a sinc function. In our case, and for the sake of simplicity, we consider an infinite antenna, and $f-k$ transform is

$$
P_{f k}\left(k_{r}, z_{D}, f\right)=\left|A \sum_{m=1}^{+\infty} \psi_{m}\left(z_{s}\right) \psi_{m}\left(z_{D}\right) S(f) \delta\left(k_{r m}-k_{r}\right)\right|
$$

To conclude, if the source has a constant spectrum $S$, which is the case in our study, we obtain (4)

$$
P_{f k}\left(k_{r}, z_{D}, f\right)=\left|B \sum_{m=1}^{+\infty} \psi_{m}\left(z_{s}\right) \psi_{m}\left(z_{D}\right) \delta\left(k_{r}-k_{r m}\right)\right|,
$$

where $B=A * S$ is a constant.

\section{ACKNOWLEDGMENTS}

The authors thank Dr. P. Roux and Pr. W. A. Kuperman at Marine Physical Laboratory (SCRIPPS Institution of Oceanography, San Diego) for the ultrasonic experiments and Compagnie Générale de Géophysique for providing at sea data. Most of the computations presented in this paper were performed at the Service Commun de Calcul Intensif de l'Observatoire de Grenoble (SCCI) using the modeling software of J. Virieux and S. Operto (Laboratoire Géosciences Azur, Nice). This work has been partially supported by the Délégation Générale pour l'Armement (DGA) and followed by D. Fattaccioli.

\section{REFERENCES}

[1] H. P. Bucker, "Use of calculated sound fields and matched-field detection to locate sound sources in shallow water," The Journal of the Acoustical Society of America, vol. 59, no. 2, pp. 368373, 1976.

[2] D. F. Gingras, "Robust broadband matched-field processing: performance in shallow water," IEEE Journal of Oceanic Engineering, vol. 18, no. 3, pp. 253-264, 1993.

[3] R. K. Brienzo and W. S. Hodgkiss, "Broadband matched-field processing," The Journal of the Acoustical Society of America, vol. 94, no. 5, pp. 2821-2831, 1993.

[4] J. A. Fawcett, M. L. Yeremy, and N. R. Chapman, "Matchedfield source localization in a range-dependent environment," The Journal of the Acoustical Society of America, vol. 99, no. 1, pp. 272-282, 1996.

[5] C. W. Bogart and T. C. Yang, "Source localization with horizontal arrays in shallow water: spatial sampling and effective aperture," The Journal of the Acoustical Society of America, vol. 96, no. 3, pp. 1677-1686, 1994.

[6] A. B. Baggeroer, W. A. Kuperman, and P. N. Mikhalevsky, "An overview of matched field methods in ocean acoustics," IEEE Journal of Oceanic Engineering, vol. 18, no. 4, pp. 401-424, 1993.

[7] E. C. Shang, "Source depth estimation in waveguides," The Journal of the Acoustical Society of America, vol. 77, no. 4, pp. 1413-1418, 1985.

[8] T. C. Yang, "A method of range and depth estimation by modal decomposition," The Journal of the Acoustical Society of America, vol. 82, no. 5, pp. 1736-1745, 1987.

[9] C. W. Bogart and T. C. Yang, "Comparative performance of matched-mode and matched-field localization in a rangedependent environment," The Journal of the Acoustical Society of America, vol. 92, no. 4, part 1, pp. 2051-2068, 1992.

[10] J. F. Lynch, S. D. Rajan, and G. V. Frisk, "A comparison of broadband and narrow-band modal inversions for bottom geoacoustic properties at a site near Corpus Christi, Texas," The Journal of the Acoustical Society of America, vol. 89, no. 2, pp. 648-665, 1991.

[11] G. R. Potty, J. H. Miller, J. F. Lynch, and K. B. Smith, “Tomographic inversion for sediment parameters in shallow water," The Journal of the Acoustical Society of America, vol. 108, no. 3 , pp. 973-986, 2000.

[12] G. V. Frisk and J. F. Lynch, "Shallow water waveguide characterization using the Hankel transform," The Journal of the Acoustical Society of America, vol. 76, no. 1, pp. 205-216, 1984.

[13] S. D. Rajan, J. F. Lynch, and G. V. Frisk, "Perturbative inversion methods for obtaining bottom geoacoustic parameters in shallow water," The Journal of the Acoustical Society of America, vol. 82, no. 3, pp. 998-1017, 1987.

[14] I. Tolstoy and C. S. Clay, Ocean Acoustics, AIP Press, New York, NY, USA, 1987.

[15] B. Nicolas, J. I. Mars, and J.-L. Lacoume, "Geoacoustical parameters estimation with impulsive and boat-noise sources," 
IEEE Journal of Oceanic Engineering, vol. 28, no. 3, pp. 494 $501,2003$.

[16] E. C. Shang, C. S. Clay, and Y. Y. Wang, "Passive harmonic source ranging in waveguides by using mode filter," The Journal of the Acoustical Society of America, vol. 78, no. 1, pp. 172$175,1985$.

[17] G. R. Wilson, R. A. Koch, and P. J. Vidmar, "Matched mode localization," The Journal of the Acoustical Society of America, vol. 84, no. 1, pp. 310-320, 1988.

[18] R. T. Kessel, "Extracting modal wave numbers from data collected in range-dependent environments," The Journal of the Acoustical Society of America, vol. 104, no. 1, pp. 156-162, 1998.

[19] J. Virieux, "P-SV wave propagation in heterogeneous media: velocity-stress finite-difference method," Geophysics, vol. 51, no. 4, pp. 889-901, 1986.

[20] J.-L. Mari, G. Arens, D. Chapellier, and P. Gaudiani, Geophysics of Reservoir and Civil Engineering, Editions Technip, Paris, France, 1999.

[21] S. M. Jesus, "Normal-mode matching localization in shallow water: environmental and system effects," The Journal of the Acoustical Society of America, vol. 90, no. 4, pp. 2034-2041, 1991.

[22] N. E. Collison and S. E. Dosso, "Regularized matched-mode processing for source localization," The Journal of the Acoustical Society of America, vol. 107, no. 6, pp. 3089-3100, 2000.

[23] B. Nicolas, "Identification du milieu océanique et localisation de source en ultra basse fréquence (1-100 Hz)," Ph.D. dissertation, INPG, Grenoble, France, 2004.

[24] R. C. Weast, Handbook of Chemistry and Physics, CRC Press, Boca Raton, Fla, USA, 1984.

Barbara Nicolas graduated from the Ecole Nationale Supérieure des Ingénieurs Electriciens de Grenoble, France, and received the M.S. and Ph.D. degrees in signal processing from the National Polytechnic Institute of Grenoble (INPG), France, respectively, in 2001 and 2004. In 2005, she began a postdoctoral appointment at the French Atomic Energy Commission (C.E.A.) in medical imaging. Her research interests in-

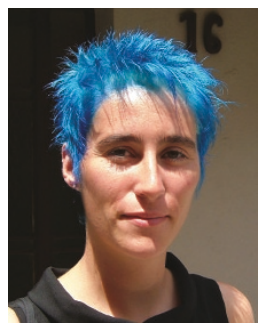
clude geoacoustic inversion and localization in underwater acoustics and medical imaging.

Jérôme I. Mars received the M.S. degree in 1986 in goephysics from the Joseph Fourier University, Grenoble, France, and the $\mathrm{Ph} . \mathrm{D}$. in signal processing in 1988 from the National Polytechnic Institute of Grenoble, France. From 1989 to 1992, he was a postdoctoral researcher at the Centre des Phénoménes Aléatoires et Géophysiques, Grenoble, France. From 1992 to 1995, he was a Visiting Lecturer and Scientist in the Materials Sciences and Mineral Engineering Department, University of California, Berkeley. He is currently a Professor in signal processing at the National Polytechnic Institute of Grenoble (INPG) and he works for the Laboratoire des Images et des Signaux. His research interests include seismic and acoustic signal processing, wavefield separation methods, time-frequency time-scale characterization, and applied geophysics.

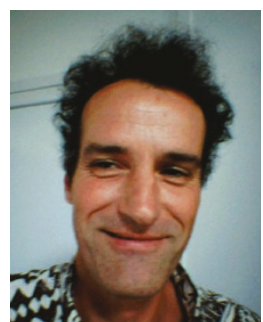

Jean-Louis Lacoume graduated from the Ecole Normale Supérieure, Paris, France, as a certified teacher in 1964 and as a Docteur d'état in 1969 . He was a Lecturer in physics at Orsay University, Orsay, France, from 1964 to 1967 and in geophysics at Paris VI University from 1969 to1972. He has been a Professor at the National Polytechnic Institute of Grenoble (INPG), France, since 1972 where he studied waves physics

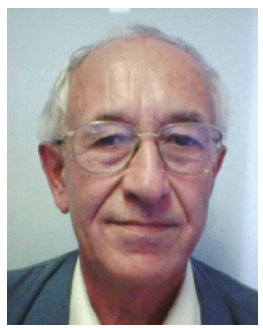
and signal processing. He has published seven books and 50 papers on reviews and 110 communications on conferences. His research and teaching activities are in wave physics and signal processing: electromagnetic waves in the earth environment, acoustic and elastic waves in the earth and the oceans, vibrations of mechanical systems, spectral and cross-spectral analysis, and higher order statistics. He was the Director of the Research Center on Random Phenomena and Geophysics, Grenoble, France, from 1972 to 1989, Dean of the Post-Graduate School at INPG from 1989 to 1995, and Director of the Laboratoire des Images et des Signaux, Grenoble, France, from 1998 to 2000. 